University of Louisville

ThinkIR: The University of Louisville's Institutional Repository

Electronic Theses and Dissertations

8-2012

\title{
Individual and developmental differences in susceptibility to the irrelevant speech effect.
}

Amanda Frances O'Bryan

University of Louisville

Follow this and additional works at: https://ir.library.louisville.edu/etd

\section{Recommended Citation}

O'Bryan, Amanda Frances, "Individual and developmental differences in susceptibility to the irrelevant speech effect." (2012). Electronic Theses and Dissertations. Paper 1077.

https://doi.org/10.18297/etd/1077

This Doctoral Dissertation is brought to you for free and open access by ThinkIR: The University of Louisville's Institutional Repository. It has been accepted for inclusion in Electronic Theses and Dissertations by an authorized administrator of ThinkIR: The University of Louisville's Institutional Repository. This title appears here courtesy of the author, who has retained all other copyrights. For more information, please contact thinkir@louisville.edu. 
INDIVIDUAL AND DEVELOPMENTAL DIFFERENCES IN SUSCEPTIBILITY TO THE IRRELEVANT SPEECH EFFECT

\title{
By
}

Amanda Frances O'Bryan

B.S., College of Charleston, 1999

M.S., University of Louisville, 2009

\begin{abstract}
A Dissertation
Submitted to the Faculty of the

College of Arts and Sciences of the University of Louisville In Partial Fulfillment of the Requirements

For the Degree of
\end{abstract}

Doctor of Philosophy

Department of Psychological and Brain Sciences

University of Louisville

Louisville, Kentucky

August, 2012 


\section{Copyright 2012 by Amanda Frances O’Bryan}

All rights reserved 
Individual and Developmental Differences in Susceptibility to the Irrelevant Speech Effect

\author{
By
}

Amanda Frances O'Bryan

B.S., College of Charleston, 1999

M.S., University of Louisville, 2009

A Dissertation Approved on

July $17^{\text {th }}, 2012$

By the following Dissertation Committee:

Pavel Zahorik, Dissertation Director

Keith Lyle

Ed Essock

David Pisoni

Cara Cashon 


\section{DEDICATION}

This dissertation is dedicated to my daughter. She makes everyday seem like a gift, and inspires me to be the best possible person I can. This entire process was easier knowing that I would always see her smiling face when I got home. 


\section{ACKNOWLEDGEMENTS}

First, I would like to thank my committee members; Pavel Zahorik, Keith Lyle, Cara Cashon, Ed Essock, and David Pisoni. Each of you has been instrumental in helping me through this entire process. Thank you for your great questions and insights. I would like to especially thank my mentor, Pavel Zahorik. Pavel, your generosity and kindness extends above and beyond what is required of a mentor. You create an engaging and exciting work environment, and motivate all who work for you to do the best they possibly can. You are an inspiration to me as a mentor, as well as a teacher.

I would like to thank my lab members, both current and past. Gina Collechia and Chris Wheeler, thanks for making sure things were running smoothly. Derek Knisely, you were the best lab assistant, data collector and kid wrangler anyone could ask for! And Bethany Moore, your early assistance with the beginnings of the creation of the project was invaluable. Also, Ann Rothpletz, you've been a wonderful mentor as well. I'd also like to thank my fellow grad student lab partners, Gene Brandewie, Paul Anderson, and Carissa Shafto. I will miss you guys, and I hope that you go on to do incredible things! I'd also like to thank all the families that participated in the research.

Finally, I'd like to thank my incredible husband, Chris. This will always go down in our history as the craziest year ever. You always know when I need a push, and when to have patience. You're my best friend, and the best father our daughter could have, and I really couldn't have done this without you. 


\begin{abstract}
INDIVIDUAL AND DEVELOPMENTAL DIFFERENCES IN SUSCEPTIBILITY TO THE IRRELEVANT SPEECH EFFECT

Amanda Frances O'Bryan
\end{abstract}

$7 / 17 / 2012$

The Irrelevant Speech Effect (ISE) is a phenomenon in which the presentation of auditory materials (typically speech) impairs the serial recall of visually presented materials, either digits or letters. Although this effect has been replicated in a large number of studies, a wide range of individual differences in susceptibility to the auditory distracters has been found. The current study investigates four possible determinants of individual differences of the ISE: (1) The sequence length of the short-term memory (STM) task, (2) the content of the STM task and auditory distracter, (3) the STM capacity of the individual, and (4) developmental differences.

Results from a series of experiments suggest that (1) increasing sequence length can have substantial negative effects on STM task performance, but it does not increase the ISE. This indicates that individual differences in the ISE do not depend on individual differences related to task difficulty. (2) When sequence length is adjusted to equate STM task performance, the ISE is only observed for speech distracters. This result is important because it implies a primary role of phonological rehearsal in the ISE. Additional testing with a visuo-spatial STM task that did not require rehearsal showed a release from the ISE. (3) Individuals with high memory capacity appear to be less 
susceptible to the ISE. This result is among the first to demonstrate the roll of STM capacity in the ISE, and is consistent with past work that shows relationships between working memory capacity (WMC) and susceptibility to distraction. (4) When tested at span (the longest sequence length an individual can recall $50 \%$ of the time), children and adults show similar magnitudes of the ISE. This too is consistent with the view that the ISE is related to memory capacity, rather than age.

Taken together, these outcomes indicate that the ISE is caused by a disruption of memory encoding at the phonological rehearsal stage, which in turn is related to memory capacity. These results inform theory development of STM and the processes involved in auditory attention. In addition, results may be applied to the improvement of acoustical conditions in learning environments. 


\section{TABLE OF CONTENTS}

CHAPTER

PAGE

Dedication ............................................................................................ ii

Acknowledgements.................................................................................. iv

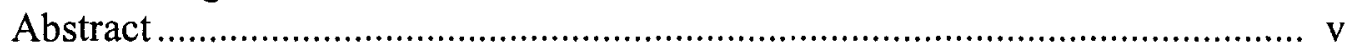

List of Figures ..................................................................................... viii

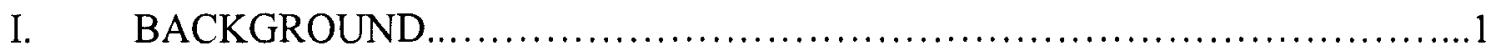

ISE Process or Content? .......................................................................

Development and the Importance of Attention.......................................

Memory Capacity and the ISE ..........................................................14

Predictions..................................................................................18

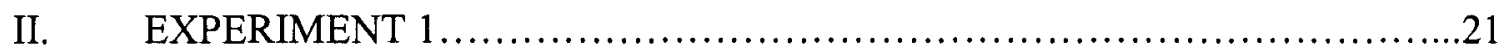

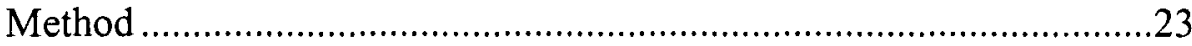

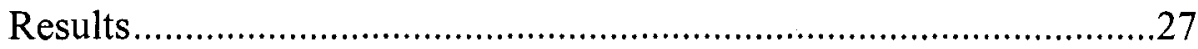

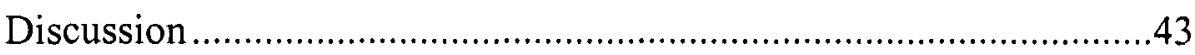

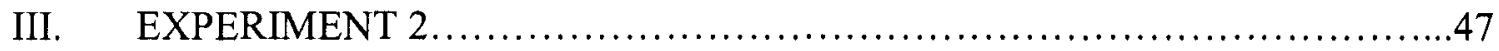

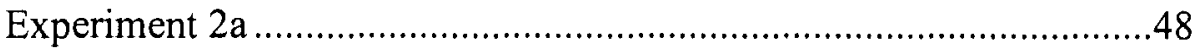

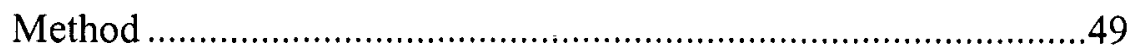

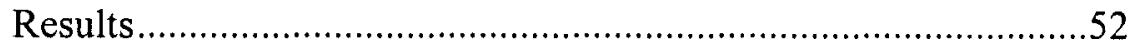

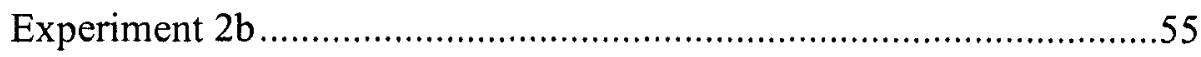

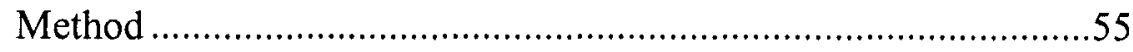

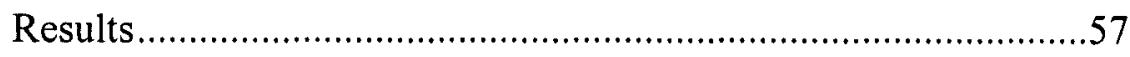

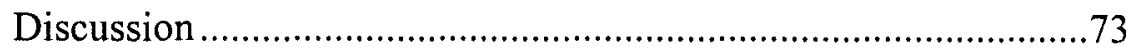


IV. GENERAL DISCUSSION..........................................77

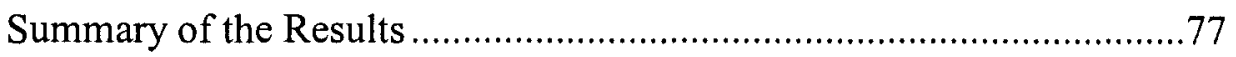

Interpretations with Regard to Current Theory.....................................8 82

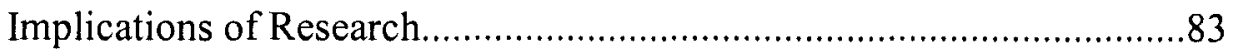

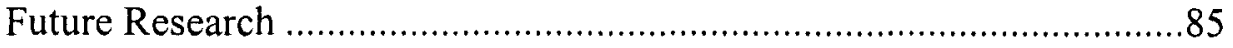

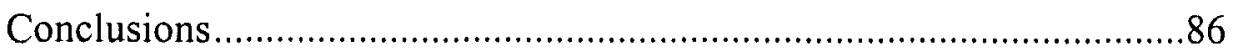

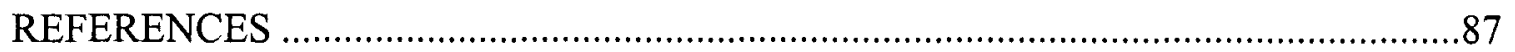

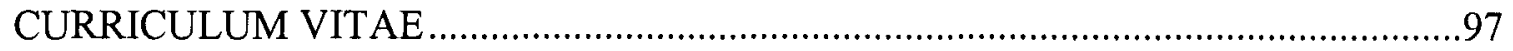




\section{LIST OF FIGURES}

$\begin{array}{lll}\text { Figures } & \text { Page }\end{array}$

1.1. Schematic of the Working Memory Model 2

2.1. Details of Experimental Procedure 26

2.2. Examples of Individual Participants' ISE data 30

2.3. Span Level Data for Child Participants 31

2.4. Span Level Data for Adult Participants 32

2.5. Distribution of Spans 33

2.6. Results of Serial Recall Task with Auditory Distraction 34

2.7. Results of Serial Recall Task by Age Group 36

2.8. Span Groups by Age $\quad 39$

2.9. Serial Position Curves for Child Span Groups 40

2.10. Serial Position Curves for Adult Span Groups 41

3.1. Schematic of the Visuo-spatial Serial Recall Task 53

3.2. Results from Serial Recall Task with AS 54

3.3. Distribution of Digit Span Data 59

3.4. Distributions of Visuo-spatial Span Data 60 
3.5. Relationship Between Visuo-spatial Span and Digit Span 62

3.6. Mean Percent Correct for the Two Memory Tasks 64

3.7. Results of Digits Task by Age 66

3.8. Results of Visuo-spatial Task by Age $\quad 67$

3.9. Span Group Data for Digit Span Task 71

3.10. Span Group Data for Visuo-spatial Span Task 74

4.1 Child Span Distributions for the Two Experiments 80 


\section{CHAPTER I}

\section{BACKGROUND}

In a learning environment, such as a classroom, there is often auditory noise not relevant to the learning task. Occasionally, a student may not be able to inhibit this incoming auditory distraction and their performance on a visual learning task will suffer. The magnitude of a crossmodal interaction of this kind can vary with the type of noise and the individual's capability to ignore the noise. Although the effects of chronic noise exposure on learning have been documented, such as the consequences of having a school next to an airport, less is known about the effects of these kinds of brief interruptions of learning. The Irrelevant Speech Effect (ISE) is a paradigm frequently used to study the effects of irrelevant auditory input on visual learning. The ISE is used to examine the effects of specific types of noise (speech versus nonspeech) on a specific cognitive process, the retention of materials in short-term memory.

\section{ISE: Content or Process?}

The origin of the ISE can be traced to observations made by Colle and Welsh (1976). Their study was designed to test for the phonological similarity effect, an effect in which recall on a visual short-term memory (STM) task is disrupted if the items on the 
list are phonologically similar to one another (Baddeley, 1972). For example, if the tobe remembered list contains words that rhyme, an individual will exhibit a greater number of errors at recall. Colle and Welsh (1976) tested this effect using an auditory noise distracter (speech or white noise) in order to investigate whether the effect was multimodal. They discovered that the speech distracters caused a greater number of errors than did the white noise. They attributed this to the phonological similarity of the distracter (continuous speech) and the to-be-remembered items (a list of consonant letters). Later Salamè and Baddeley (1982) found similar results and termed this phenomenon the irrelevant speech effect. These investigators concluded that there may be a phonological memory store which encodes auditory speech information in the same location as visual verbal information (Baddeley, 1983).

Based on this and other evidence for phonological coding in memory, Baddeley introduced the concepts of the articulatory loop and the phonological store to his now well-known model of working memory (Baddeley, 1983). The Working Memory Model consists roughly of three systems: the central executive, the visuo-spatial scratch-pad and the phonological store (see Figure 1.1 for a schematic of the model). The central executive is considered to be a limited capacity attention system which selects and controls processes and strategies. It supervises the two "slave systems": the articulatory loop and the visuo-spatial scratch-pad. As its name suggests, the visuo-spatial scratchpad is responsible for creating and maintaining visual information. It has been hypothesized that the visuo-spatial scratch-pad is made up of two separate mechanisms: a temporary store for visual forms and a temporary store for spatial information (Logie \& Pearson, 1997). The articulatory loop is responsible for translating visual-verbal material, 


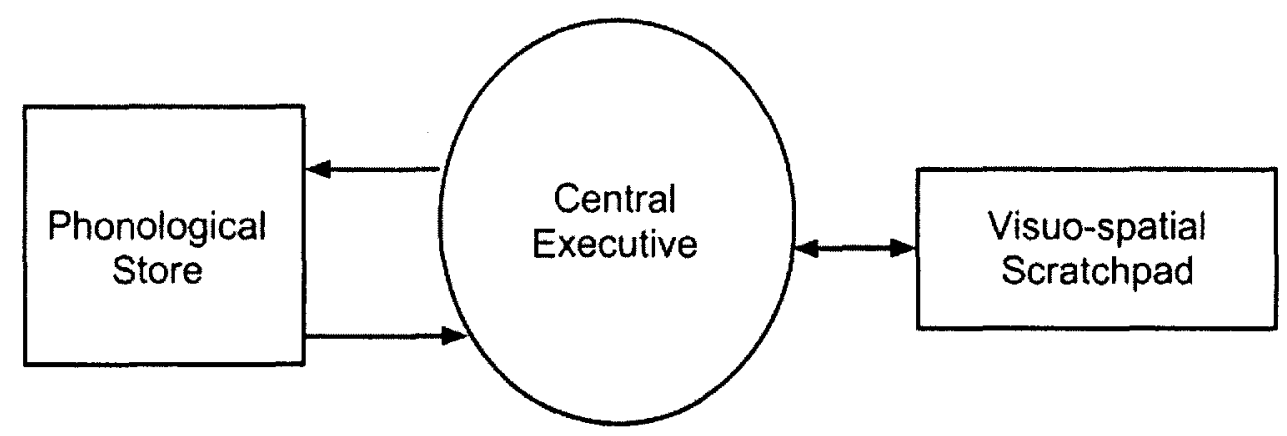

Figure 1.1. Schematic of the Working Memory Model adapted from "Working Memory," by A. D. Baddeley, 1983, Philosophical transactions of the royal society of London. Series B, Biological Sciences, 302 p. 315. 
using subvocal rehearsal, into a phonological code that is then maintained in the phonological store. Items in the phonological store are thought to begin to decay after a few seconds unless the materials are "refreshed" by the subvocal rehearsal of the articulatory loop. In addition to the translated visual material, the phonological store model posits automatic access of any auditory-verbal material, and non-access of any nonverbal materials, either auditory or visual.

According to the Working Memory Model, the ISE is attributed to the phonological similarity of the irrelevant items and the to-be-remembered items (Salamé \& Baddeley, 1982). Because auditory-verbal materials gain automatic access to the phonological store, it is speculated that these materials interfere with the visual stimuli, which must be translated into a phonemic code and are also represented in the phonological store. The increase in errors occurs because the auditory phonemes and the to-be-remembered visual stimuli are encoded similarly, thus causing confusion. Salamè and Baddeley demonstrated that as phonological similarity between the relevant and irrelevant stimuli increase, the degree of interference also increases. The phonological similarity effect, taken together with the word length effect and the articulatory suppression effect lent support for the existence of two memory systems, one based on phonological coding and accessible through either audition or articulation (the articulatory loop), and a second store used for nonverbal visual material (the visual- spatial scratch-pad) (Baddeley, 1983).

Several subsequent experiments (Bridges \& Jones, 1996; Jones \& Macken, 1995; LeCompte \& Shaibe, 1997) failed to replicate the effects of phonological similarity on the ISE found in Salamè and Baddeley's (1982) study. Thus, Jones and 
colleagues hypothesized that the ISE may be attributed instead to a preattentive process of segmentation, rather than phonological similarity between the visual target and auditory distracter (Jones, Macken, \& Murray, 1993). They stated that this preattentive process automatically segments the auditory materials into units and organizes them. The occurrence of the ISE is the result of a confusion of this automatic ordering process of the auditory materials and the necessary ordering of the visual materials. This hypothesis would predict a greater ISE for auditory distracters that require ordering, such as speech than for than for auditory distracters that do not require ordering, such as white noise (Jones et al., 1990).

In a series of experiments designed to test this hypothesis these investigators examined the ISE using a class of non-speech sounds, specifically "glissandi" (frequency glides) as auditory distracters. In the first experiment they measured the ISE using continuous and interrupted glissandi, and found that the interrupted stimuli created a significant ISE, whereas the continuous glissandi resulted in no significant ISE. They then tested the effects of embedding the glissandi with a low pass noise that was the same bandwidth and frequency range as the glide. This manipulation resulted in no significant ISE, presumably because it provided a perceptual continuity in which listeners would "fill in" the missing parts of the glide and thus create the illusion of a continuous glide. In contrast, glissandi embedded with a high pass noise gave the impression of an interrupted glide and resulted in a significant ISE (Jones et al., 1993).

This collection of experiments initiated an alternative hypothesis to explain the occurrence of the ISE; Jones termed this the changing state hypothesis (Jones, Madden \& Miles, 1992). Changing state is described as the "registration of mismatch 
between successive, segmented entities in the auditory stream" (Macken, Mosdell, \& Jones, 1999, p. 810). When such a mismatch occurs, the serial ordering of those entities is automatically encoded. According to this hypothesis, changing state auditory input is encoded in memory as sequences of discrete "objects" connected by cues to a serial order. The interference with a visual serial recall task caused by changing-state irrelevant sound results from the "conflict in memory between the order cues represented in the auditory stream and those being rehearsed in the to-beremembered stream" (Macken et al., 1999, p. 810).

According to the changing state hypothesis, the ISE is driven by the unpredictable nature of the auditory distracter, rather than its content. Thus speech and non-speech distracters are viewed as functionally equivalent (Jones \& Macken, 1993). Non-speech sounds can still cause an ISE as long as they meet the requirement for changing state. This proposal was in direct contrast to Baddeley's model which assumed a separate storage for phonological materials, and a lack of ISE for non-speech materials. Jones and colleagues hypothesized that there was instead a unitary storage which was accessible by either speech or non-speech materials, as well as either visual or auditory materials.

This claim was supported by a number of studies from Jones's lab showing that the phonological component of the distracter can be held constant, but by changing the perceptual organization significant disruption still occurs. For example, presenting the consonants $\mathrm{x}, \mathrm{y}$, and $\mathrm{z}$ diotically created disruption, but when they were presented stereophonically, such that ' $x$ ' is presented to one ear, ' $y$ ' to another, and ' $z$ ' to both, the perception of three separate streams was created. Each of these three separate streams 
was perceived as steady state repetitions of the three letters, and no longer caused a disruption (Jones \& Macken, 1995b). Additionally, it was demonstrated that if the content of the auditory distracter remains constant, but the timing interval between distracters changes, this also will cause an ISE (Jones \& Macken, 1995b). Furthermore, if the identity of the distracter is fixed, but the pitch is changed, there is still a strong ISE (Jones, Alford, Bridges, Tremblay, \& Macken, 1999). Finally, it was shown that two sounds changing in pitch creates an ISE, but speeding up the presentation to create a segmentation of these two sounds into two separate streams eliminates the ISE (Macken, Tremblay, Houghton, Nicholls, \& Jones, 2003).

This claim of functional equivalence of speech and non-speech distracters was called into question by both Baddeley and Larsen (2003) and others (LeCompte, Neely, \& Wilson, 1997). The ISE has been replicated in numerous studies, with a number of different auditory distracters, including: single words (Elliott, Barrilleaux, \& Cowan, 2006), ongoing speech (Colle \& Welsh, 1976), backwards speech, and foreign language speech (Jones, Miles, \& Page, 1990). However, to date, Jones and colleagues are the only group to have shown that the ISE can occur with non-speech sounds such as tones (Jones \& Macken, 1993), while another study failed to replicate these results (LeCompte, Neeley, \& Wilson, 1997). Studies by Jones and colleagues generally employed a sequence length of seven to nine items. Baddey and Larsen (2007) contended that the phonological similarity effect diminishes with long list lengths, such as the ones used by Jones et al. In support of this, Baddeley conducted a series of experiments replicating his previous findings of the phonological similarity effect using a sequence length of six (Larsen \& Baddeley, 2003). 
As yet, the impact of the sequence length of the visual task on the ISE has not been systematically investigated. A digit span is defined as the longest list of items that a person can repeat back in correct order, immediately following presentation, on $50 \%$ of all trials. Orsini et al. (1987) collected normative data on a large group of adults $(n=1355)$ and found a mean visual digit span of $5.4(S D=.89)$. Most studies of the ISE use a digit sequence of at least seven, and some as high as nine (Ellermeier \& Zimmer, 1997). Given that for the general population, these sequence lengths would be considered quite difficult, it is possible that the task difficulty is masking the ISE effects.

One goal of the current study was to examine the relationship between the difficulty (sequence length) of the visual task, and the amount of distraction experienced. This was done by testing individuals with a number of different sequence lengths (Experiment 1). The nature of the distraction was examined by using both speech and non-speech auditory distracters (Experiment 1). If the phonological similarity effect is stronger with shorter sequences, a pattern between speech and nonspeech distracters should emerge showing less ISE by speech distracters as the sequence lengths increase.

Baddeley's Working Memory Model predicts that non-speech distracters will not have an effect on a verbal serial memory task. This model also predicts the converse, that a nonverbal serial memory task will be unaffected by a verbal auditory distracter. In order to test this hypothesis Experiment 2 employed a nonverbal visuo-spatial serial memory task. This task is similar to the well-established Corsi Blocks test, a test designed by Philip Corsi to measure short-term spatial memory (Corsi, 1972). The Corsi 
Blocks test uses a platform of nine wooden $3 \times 3 \mathrm{~cm}$ blocks fastened on a $25 \times 30 \mathrm{~cm}$ baseboard in a standard random order. The experimenter taps a sequence pattern onto the blocks which participants must then replicate. The sequence length increases each trial until the participant is no longer able to correctly replicate the pattern. However, the task used in the current study was carefully designed for the participant to be unable to assign arbitrary names to the location of the blocks. Unlike the Corsi-Blocks test the blocks will not have a fixed location. Doing so will ensure that no verbal rehearsal will take place during the test.

In Baddeley's Working Memory Model, the visuo-spatial scratch-pad and phonological loop are hypothesized to be separate processes. One method used to test this is through the use of articulatory suppression (AS). AS is the method of asking participants to say an irrelevant word or phrase aloud while completing a memory task. This repetition blocks the articulatory rehearsal process, thereby causing memory traces in the phonological loop to decay. AS has been shown to have a large effect on serial memory tasks which require verbal rehearsal such as a digit span test, but little or no effect on visuo-spatial tasks such as the Corsi-Blocks test (Farmer, Berman, \& Fletcher, 1986). A condition using AS will be included in Experiment 2 in order to replicate these findings, and extend them to a child population, as well as to demonstrate the lack of verbal rehearsal needed to complete the visuo-spatial task. According to the Working Memory Model, if a task requires no verbal rehearsal, there should be no evidence of an ISE. This prediction will be tested further by including speech and nonspeech distracters on a visuo-spatial task as well (Experiment 2). Errors caused by a speech distracter on a nonverbal serial memory task would reinforce the hypothesis of a 
unitary, multimodal storage system that is described by the changing state theory.

\section{Development and the Importance of Attention}

Recently, a small number of studies have investigated the ISE in children (Elliott, 2002; Elliott \& Cowan, 2005; Klatte, Meis, Sukowski, \& Schick, 2007; Klatte, Lachmann, Schlittmeier, \& Hellbrück, 2010). A distinction of these studies from the previous work is their emphasis on the role of attention. Prior to the investigations of the ISE in children, a new theory of working memory had begun to emerge which placed far greater importance on the role of attention than did Baddeley's model (Cowan, 1995). The researchers interested in the development of the ISE stated that because the current theories, (i.e. the changing state theory and Baddeley's Model of Working Memory) assume automatic access of auditory materials and no mediation of attention, they offer no explanation for a developmental effect (Elliott, 2002; Klatte et al., 2010). They contend that the difference in the ISE found between children and adults occurs because of a developmental difference in attention.

Recently, many researchers have begun to posit that the central executive component of the Working Memory Model mediates attention (Engle, 2002; Cowan, 2005). Engle and Cowan both argue that the attention component is limited in capacity, and that rehearsal strategies may deplete the capacity for attention. Thus, if an individual is particularly challenged by a rehearsal strategy, they may be more susceptible to distraction. One could argue that some children may be more challenged by particular rehearsal strategies, because they have not yet become automatic. By this account, children would be more susceptible to distraction, and therefore show a 
greater ISE.

Since the changing state hypothesis makes no predictions per se about the development of the effects of irrelevant speech, one must speculate predictions based on this theory. It has been shown that younger children have more difficulty in their ability to differentiate between two stimulus streams (Doyle, 1973; Lane \& Pearson, 1982). Given that the changing state hypothesis places importance on the ability of streaming the stimuli (Jones, 1993), children may be more impaired by irrelevant speech than adults.

The two studies that have investigated the ISE in children found conflicting results (Elliott, 2002; Klatte, et al., 2010). Elliot's study consisted of 96 children ages 8-11, and 32 adults. They were each given a standardized digit span test prior to administration of the ISE task, to determine each participant's digit span. They were then tested with a digit span task with the sequence length set at their individual span, while hearing either changing state or steady state speech (lists of different words or the same word repeated), changing state or steady state tones (tones varying in frequency or a single tone repeated), or silence. Elliott found that the very youngest age group ( 8 year-olds) exhibited a greater ISE for the changing state speech than the older children and adults. A recent study by Klatte and colleagues (2010) also investigated the ISE in children but found no such difference using similar age groups to Elliot's study.

There are a few possible explanations for the differences found between these two studies. Klatte et al. used a visual task which required the participants to memorize pictures. Elliott's study used a digit span paradigm. There is some evidence that the picture naming tasks are easier for younger children than digit naming (Case \& 
Globerson, 1974). Additionally, the presentation rate of the visual materials in the study by Klatte et al. was slower in than in Elliott's study. There is evidence showing that children can benefit from a slower presentation rate on a task such as this (Dempster, 1981). Although there were differences between the two studies in children's errors during the condition with a speech distracter, the children's scores in quiet (no distracters) were similar in both studies. This demonstrates a similar baseline performance even though the tasks and presentation were different. Thus, it is difficult to make the claim that one task was easier than the other. However, if the inhibition of irrelevant speech requires more cognitive resources, the difficulty of the task may limit the resources available, causing a greater number of errors when a distracter is present. Although these methodological differences may have had some impact on the children's performance, there is a greater concern regarding the analyses used in these studies. Generally in studies of the ISE, participants are all given 20-30 trials at a single sequence length, usually of seven or eight digits. This method is suitable for testing adults, but digit span has been shown to be reduced in children thus rendering this an inappropriate method for them. The study cited above (Orsini et al., 1987) that gathered normative data for digit span in adults also examined digit spans in children. Although the mean digit span was 4.5 digits $(S D=.9)$, there was a substantial increase in spans across age. The mean span of children aged 4 years was 3.5 digits, whereas the average span in 10 year old children was 5.5 digits. Elliott (2002) and Klatte et al. (2010) solved this problem by giving each participant a span test prior to the experiment, and then testing each person using their predetermined span. For example, a child that has a span of four would only be tested for an ISE at a sequence length of 
four. An adult however may have a span of seven, and would then be tested for an ISE using a sequence length of seven. This method created the appearance of a greater ISE in children when the data are analyzed.

Referring back again to the past studies of the ISE, the generally accepted method of analysis is to compare the mean number of errors at each serial position. These data are then entered into an ANOVA with serial position and auditory condition as the factors. This type of analysis would be impossible with a dataset such as that collected by Elliott and Klatte et al. because all participants were tested at different sequence lengths. Their solution to this problem was to calculate a percent correct for each individual. Presumably this percent correct is calculated by dividing the number of correct digits by the total number of digits, although this calculation is not described in either study. Using this method, if an individual is tested at a span of seven for five trials, they would have a maximum of 35 digits correct. If they missed 10 out of these 35 digits they would have a score of $71 \%$ correct. However, if a child is tested at a span of four for five trials, they would have a maximum of only 20 digits. The same number of errors (10) would render them a score of $50 \%$. The potential problem comes when comparing these scores across age. Children will inevitably have fewer total digits because of their reduced spans. This will lead to results that show children having a reduced percent correct for the same number of errors. The method used in the current study was to count trials with errors at any point along the sequence length as an incorrect trial. With this method, children and adults had the same number of trials, and the calculation of percent of correct trials used the same denominator for both groups.

Finally, there is a critical assumption made when testing all individuals at a 
predetermined span: A child working at his/her maximum span is operating at the same level of performance as an adult working at his/her maximum span. The mechanisms which determine the developmental increase in STM span are not well understood. There is some evidence that developmental capacity differences may be a result of the speed of item identification. Significant correlations have been shown between speed of word identification and STM span (Case, Kurland, \& Goldberg, 1982). In addition, longer words yield slower reading rates and lower span scores (Baddeley, Thomson, \& Buchannon, 1975). It has also been demonstrated that if adults' item identification speed is slowed to a rate more comparable to children's they exhibit similar spans (Dempster, 1981). These findings have led researchers to theorize that faster item identification may allow for greater capacity for rehearsal. It is possible then that a child operating at her maximum span could be employing very different strategies than an adult operating at her maximum span. These strategy differences may lead to differences in susceptibility to the ISE.

The question of the developmental differences of susceptibility to the ISE is an important one. Research has shown that children show greater auditory distraction than adults (Gomes, Molholm, Christodoulou, Ritter, \& Cowan, 2000). Additional research supports the hypothesis that children have reduced selective attention abilities, and that these differences may arise from children's inability to inhibit the processing of irrelevant stimuli (Lane \& Pearson, 1982). Because studies of the ISE allow for a better understanding of the mechanisms of crossmodal selective attention, it can be useful to study the effects of noise on attention in children. A goal of the current study was to examine how the difficulty of the task affects children's ability to inhibit irrelevant 
sounds. This study also systematically tested whether children experience different disruption between speech and non-speech sounds, and did so while testing all individuals at the same level of performance, a variable that was not well controlled-for in other studies. Finally, differences in susceptibility to irrelevant speech between children and adults were investigated using a visuo-spatial task that requires no item identification. Use of this task removed the possible confounds of differences in capacity resulting from rehearsal strategies.

\section{Memory Capacity and the ISE}

In addition to possible developmental differences in the susceptibility to the ISE, there are large individual differences as well. Ellermeier and Zimmer (1997) used the ISE paradigm to investigate the relationship between objective and subjective measures of disruption by environmental noise. They reported individual differences in susceptibility to the ISE, a result that had not yet been reported in the literature. Their study used a typical methodology, testing all individuals $(N=72)$ with a sequence length of nine digits, under auditory conditions of quiet, speech and pink noise (a signal in which each octave carries an equal amount of noise power). They compared ISEs across individuals by calculating difference scores between the numbers of errors found in the speech condition and the number of errors in the quiet condition. The differences they found were quite large, ranging from a minimum of -18 to a maximum of +65 . These difference scores roughly correspond to a reduction of errors under the speech condition, and a very large increase of errors, respectively (Ellermeier \& Zimmer, 1997). The difference scores were fairly evenly distributed, and approximately $1 / 8$ of the participants 
experienced no ISE at all. To date, this is the only study which has reported a distribution of individual differences in the ISE.

The few remaining studies that have investigated individual differences in susceptibility to the ISE have done so by attempting to correlate these differences with an underlying cognitive construct, working memory capacity (WMC) (Beaman, 2004; Elliott \& Cowan, 2005). These studies were prompted by another study that showed a strong relationship between working memory capacity (WMC) and auditory distraction (Conway, Cowan \& Bunting, 2001). Specifically, this study demonstrated that individuals with a reduced WMC were more likely to hear their own names on a dichotic shadowing task. They hypothesized that individuals with reduced WMC are more likely to experience interference from auditory distraction during a selective attention task. WMC has also been shown to have the same relationship with other selective attention tasks such as an antisaccade task (Kane, Bleckley, Conway, \& Engle, 2001), as well as the Stroop task (Kane \& Engle, 2003).

Studies that have attempted to find a similar relationship between WMC and the ISE have reported mixed results. Beaman (2004) correlated mean number of errors on a test of ISE and performance on the Operation Span (OSPAN) task (Turner \& Engle, 1989), the main WMC test used in the studies cited above. No correlation was found between WMC and the effect of irrelevant speech. However, using the error data Beaman did show that individuals with lower WMCs included a greater number of intrusions from semantically related speech than those with higher WMCs. Those individuals with lower capacities were less able to filter out those auditory distractions that were semantically similar to the to-be-remembered items. In contrast, Elliott and 
Cowan (2005), also using the OSPAN, found a negative correlation between WMC and ISE, such that individuals with higher capacities were more affected by the irrelevant sounds.

It is important to note the potential differences between STM and working memory. Some researchers have defined the mechanism of STM as encapsulated in the process of rehearsal and storage within the two sub-systems of Baddeley's Working Memory Model (Cowan, 2008). Working memory on the other hand is considered a combination of systems in which information stored in the STM is manipulated and updated for further use, and thus requires the central executive (Baddeley, 1983). Put in other words, working memory tasks require active selective attention while STM tasks do not.

Researchers that have linked WMC to selective attention tasks claim that capacity is a direct measure of an individual's ability to inhibit distracting information (Engle, 2002). Engle has also shown using structural equation modeling that STM capacity, while correlated with WMC, is best modeled as a distinct construct (Engle, Tuholski, Laughlin, \& Conway, 1999). The current theories of the ISE argue that the auditory materials enter into the processing stream automatically, with no role for attention. If WMC tasks are actually a measure of attention, as Engle argues, then neither Baddeley's model, nor the changing state hypothesis would predict that WMC would be related to susceptibility to the ISE. While measures of WMC may not be predictive of the ISE, it is also important to rule out measures of STM capacity.

The study discussed above (Ellermeier \& Zimmer, 1997) is also the only study which has attempted to correlate STM capacity with susceptibility to irrelevant sounds. 
Of importance to this proposed research is Ellermeier and Zimmer's statement that the individual effect sizes were not correlated with the capacities of the individuals. They correlated the effect sizes with the number of digits recalled in a noise condition. This analysis is problematic for two reasons. First, Ellermeier and Zimmer tested all subjects at a sequence length of nine digits. For some individuals this may be well beyond their STM capacity. Using this method, the determination of span based on their error rate at a long sequence length is more comparable to a supraspan test, in which individuals are tested with long sequence lengths and required to recall as many digits as they can. It has been shown that supra-span scores and serial recall scores are not well correlated and may reflect different processes (Dempster, 1981). Second, error rate in the noise condition was assumed to be indicative of an individual's typical error rate in quiet, since the two conditions had roughly the same average number of errors. But as stated by Ellermeier and Zimmer, taking an average across participants could be misleading because of the amount of variability of the effect across individuals. It is not clear if the variance of error rate in the noise condition was similar to the variance in the quiet condition.

As yet, the evidence is inconclusive whether STM capacity is at all related to susceptibility to the ISE. There are good reasons to continue to pursue this question. Researchers who have investigated developmental differences of the ISE argue that the greater susceptibility found in children results from developmental attentional differences (Elliott, 2002; Elliott \& Cowan, 2005). If this is the case there should be a correlation between attentional measures (WMC) and susceptibility to the ISE, a finding that has yet to be supported (Beaman, 2004). Furthermore, the changing state theory, which does not posit a role of attention in the ISE, would predict no influence of the role of capacity on 
the ISE. The current study addressed these issues by testing the relationship between STM capacity and the ISE directly, in both children and adults.

\section{Predictions}

Based on this review of the literature, Baddeley's Model of Working Memory appears to provide a better explanation for the ISE. Historically, the model has been successful at explaining why memory capacity is related to attention switching or the ability to inhibit distraction (Conway et al., 2001; Kane \& Engle, 2001; Kane et al., 2001). Because the ISE is also a measure of the ability to inhibit distraction, one would it expect that it too could be explained by the Working Memory Model. In contrast, the changing state theory assumes automatic access of all incoming visual and auditory materials, and thus allows for no role for attention. In addition, the changing state theory has been limited primarily to findings that relate to the ISE, but has not yet been applied to other tests of attention.

The changing state theory posits a multimodal processing strategy, such that visual and auditory materials, both verbal and nonverbal, are all processed similarly. However, there is substantial evidence that visuo-spatial and phonological materials are processed separately (Baddeley, 1986; Farmer et al., 1986; Logie, 1986). In addition, there is some evidence that speech distracters in particular cause greater amounts of distraction on visual STM tasks (LeCompte, et al., 1997). Nonetheless, proponents of the changing state theory have challenged the hypothesis of a special role for speech distracters with regards to the ISE (Tremblay, Nichols, Alford \& Jones, 2000).

The changing state theory has also not addressed the question of the development 
of the ISE. Because attentional resources are theorized to be the cause of greater susceptibility to the ISE in children (Elliott, 2002), the changing state theory's lack of a role for attention makes it difficult to be applied to developmental studies. In contrast, the Working Memory Model has been applied to studies investigating the inhibition of distraction in children, although not specifically the ISE. Hale, Bronik and Fry (1997) used The Working Memory Model to investigate developmental change in interference of verbal and spatial memory, and found age-dependent, domain-specific changes. To date, the changing state theory cannot fully address the developmental differences found in the ISE, or any other types of STM disruption.

Finally, the changing state theory also gives no explanation for the role of memory capacity and its relationship to the inhibition of distraction. Proponents of the Working Memory Model have posited that capacity differences may be a result of inefficiencies in rehearsal strategies. These inefficiencies may lead to a recruitment of attentional resources away from the central executive, leaving the individual more susceptible to distraction (Engle, 2002). According to the changing state theory, rehearsal strategies would play no part in the occurrence of the ISE, thus differences in capacity would not influence the amount of susceptibility.

For these reasons, the central hypothesis of this work is that the ISE may best be explained in terms of the phonological loop in Baddeley's Model of Working Memory. This model predicts:

1) Greater ISE for speech than non-speech distracters

2) Effect of speech distracter will diminish as the sequence length of the STM task increases 
3) No ISE for visuo-spatial STM tasks that do not use the phonological loop for encoding.

4) Similar ISE for children and adults, provided STM capacities are similar

5) Greater ISE for individuals with reduced STM capacity

The overall goal of this dissertation is to test these five predictions. This was accomplished in a series of three experiments $(1,2 \mathrm{a}, \& 2 \mathrm{~b})$. Prediction 1 was assessed by using both speech and non-speech auditory distracters (Experiments $1 \& 2 b$ ). Prediction 2 was evaluated by employing a method that tested individuals with a range of sequence lengths and compared performance across this range (Experiment 1). Prediction 3 was assessed by using a visuo-spatial STM task (Experiments $2 \mathrm{a} \& 2 \mathrm{~b}$ ). Prediction 4 was investigated by testing both children and adults using these methods and comparing age group performance (All Experiments). Finally, prediction 5 was addressed by examining the performance of individuals tested at span, and analyzing the difference between span groups (Experiments $1 \& 2 b)$. 


\section{CHAPTER II}

\section{EXPERIMENT 1}

In order to test the predictions made by the phonological loop theory (Baddeley, 1983 ) and the changing state theory (Jones, Macken, \& Nicholls, 2004), the sequence lengths of the visual STM task were systematically varied. Varying the length of the visual task is not the typical method used in other studies. Generally, participants are tested at one set sequence length that is predetermined by the experimenter. However, the use of a single sequence length to test all individuals does not control for the variability in STM capacity. Therefore, testing all individuals at the same sequence length creates a potential confound; individuals with low capacity may show greater errors on the trials with auditory distraction because of the difficulty of the task. Individuals with lower capacities may have fewer attentional resources, which are compromised as the task increases in difficulty. In order to ensure an equal level of performance across all participants, individuals were tested with a range of sequence lengths which was determined with the aim of spanning the entire range of that participant's performance

This method of testing at various sequence lengths also allowed an examination of developmental differences of the ISE. A secondary goal of this method was to examine 
the impact of speech and non- speech distracters in children, while controlling for the level of performance of the participant. Conflicting evidence in the literature has demonstrated both that children are more affected by speech than adults (Elliott, 2002) and that they are not distinct from adults in their susceptibility to different auditory stimuli (Klatte, et al., 2010). Previous studies have used as their dependent variable the percentage of correct digits. This percentage is calculated by dividing the number of correct digits by the total number of digits. Children have reduced STM capacity. Thus this method biases the results by consistently calculating percent correct with a smaller denominator for the children. The current study has chosen to use instead the percentage of correct trials, with the intent to remove this bias. With this method it is hypothesized that children will not show an overall increase in susceptibility to the ISE, nor will they show a different effect for speech vs. non-speech distracters.

A final aim of Experiment 1 was to examine the relationship between individual memory capacity and susceptibility to the ISE. This will also be made possible with the method of testing at various sequence lengths. A memory span was able to be extracted for each individual using the percentage of correct trials data. Span was defined as the maximum sequence length which produced performance closest to $50 \%$ correct, with no distracters. A performance level at 50\% correct is considered comparable to a span as measured on a standardized test of serial STM (Dempster, 1981). Establishing a span for each participant will allow an examination of the serial position errors at different sequence lengths, an analysis that has not been done in any previous studies of the ISE. In this way, a valid comparison can be made of serial position errors across individuals with different spans. In order for this comparison 
across serial position to be made, it must be established that all individuals are operating at a similar level of performance. By equating the individuals based on span, this comparison can now be made.

\section{Method}

Participants. Forty-nine participants were recruited using an internet news service provided by the University of Louisville (UofL Today http://louisville.edu/uofltoday). Participants were divided into two age groups. Individuals 18 years and over were considered adults and those under 18 years as children. Twenty-one children ( 9 female) participated. Ages ranged from 6.8-15.6 years $(M=10.6, S D=2.5)$. Twenty-eight adults (20 female) participated. Ages ranged from 18.5-50.12 years $(M=26.9, S D=9.9)$. All participants were required to pass a $20 \mathrm{~dB}$ $\mathrm{HL}$ audiometric screening at octave frequencies $250-8000 \mathrm{~Hz}$. All participants verbally reported normal or corrected-to-normal vision and color vision. No participants reported previously diagnosed attention problems. Participants were compensated $\$ 10.00$ an hour for their time.

Apparatus/materials. Participants were tested one at a time in a double-walled sound isolation chamber (Acoustic Systems). Each individual was presented with sounds over calibrated Beyerdynamic DT-990-Pro headphones, at a comfortable level (approximately $65 \mathrm{~dB}$ SPL).

Visual Stimulus. Software used for the visual portion of the task was written in MATLAB. The visual material to be memorized consisted of random permutations of the digits $0-9$. For sequence length ranges up to 10 , digits were not repeated. For 
ranges above 10 , digits were repeated but presentation was controlled so no two repeating digits were presented adjacent to one another. Digits were presented sequentially in the center of a monitor. The digits were white, sans serif font, and subtended a visual angle of approximately $5^{\circ}$ at the viewing location.

Auditory Stimulus. The tones were generated using a MATLAB script that constructs a diatonic scale of 8 notes from $500 \mathrm{~Hz}$ to $1000 \mathrm{~Hz}$. The tone distracters were then selected randomly from these eight notes and presented in a random order on each trial. The sampling rate was $22,050 \mathrm{~Hz}$, and the duration of each tone was $500 \mathrm{~ms}$. The time interval between tones was randomized from 0 to $1 \mathrm{~s}$ with a uniform distribution. Letters distracters were recordings of a single female talker saying nine different consonants. Recordings were made in a semi-anechoic chamber using Audition. The consonants were selected in random order, also with random interstimulus intervals between 0 and $1 \mathrm{~s}$. In a third condition, no sound was presented.

Procedure. Each trial was initiated with a $500 \mathrm{~ms}$ fixation cross at the center of the screen after which the visual stimuli began. A stream of digits was presented, each digit displayed for $500 \mathrm{~ms}$ with a $500 \mathrm{~ms}$ inter-stimulus interval. Following a $5 \mathrm{~s}$ retention interval, a keypad appeared on the screen containing the digits $0-9$. Participants then entered their response by sequentially clicking on the digits displayed. They were given the opportunity to change their answer. Response time was self-paced. Upon completion participants then clicked on a green "done" button which initiated a feedback screen. Feedback consisted of presenting the entire list of digits that the participant had entered, with either happy faces or stars representing the items they had 
correctly named, and the digits incorrectly named in red. (See Figure 2.1 for a visual example of a single trial)

Participants were given instructions which stated that they recall the digits in the order that they saw them, while ignoring any sounds presented over the headphones. They were asked not to rehearse the digits out loud, but only quietly to themselves.

Sequence length manipulation. Each participant was tested with a range of sequence lengths determined by their ability. The goal was to test sequence lengths for which their entire range of performance could be plotted. For example, if a participant was consistently responding at $100 \%$ correct for a sequence length of 4 , she was not presented lengths of 3 or 2 . Conversely, if performance levels were near chance at a length of 7 , a higher sequence length was not presented. Span was defined as the maximum sequence length which produced performance closest to $50 \%$ correct, in quiet. In general, standardized tests of digit span define span as the highest list length at which an individual can correctly replicate one out of two trials (Dempster, 1981). Thus, the proposed method of determining span in the current study, while not typical, is consistent with a well-accepted method.

A single run consisted of 20-25 trials of varying sequence lengths, with five trials at each length. Sequence lengths were randomly presented within runs. Initially all participants were tested with a maximum of 10 digits. However, it became apparent that a 10 digit maximum was insufficient to describe the performance of certain participants. These three participants were re-tested with longer sequence lengths, ranging up to 20 digits. 


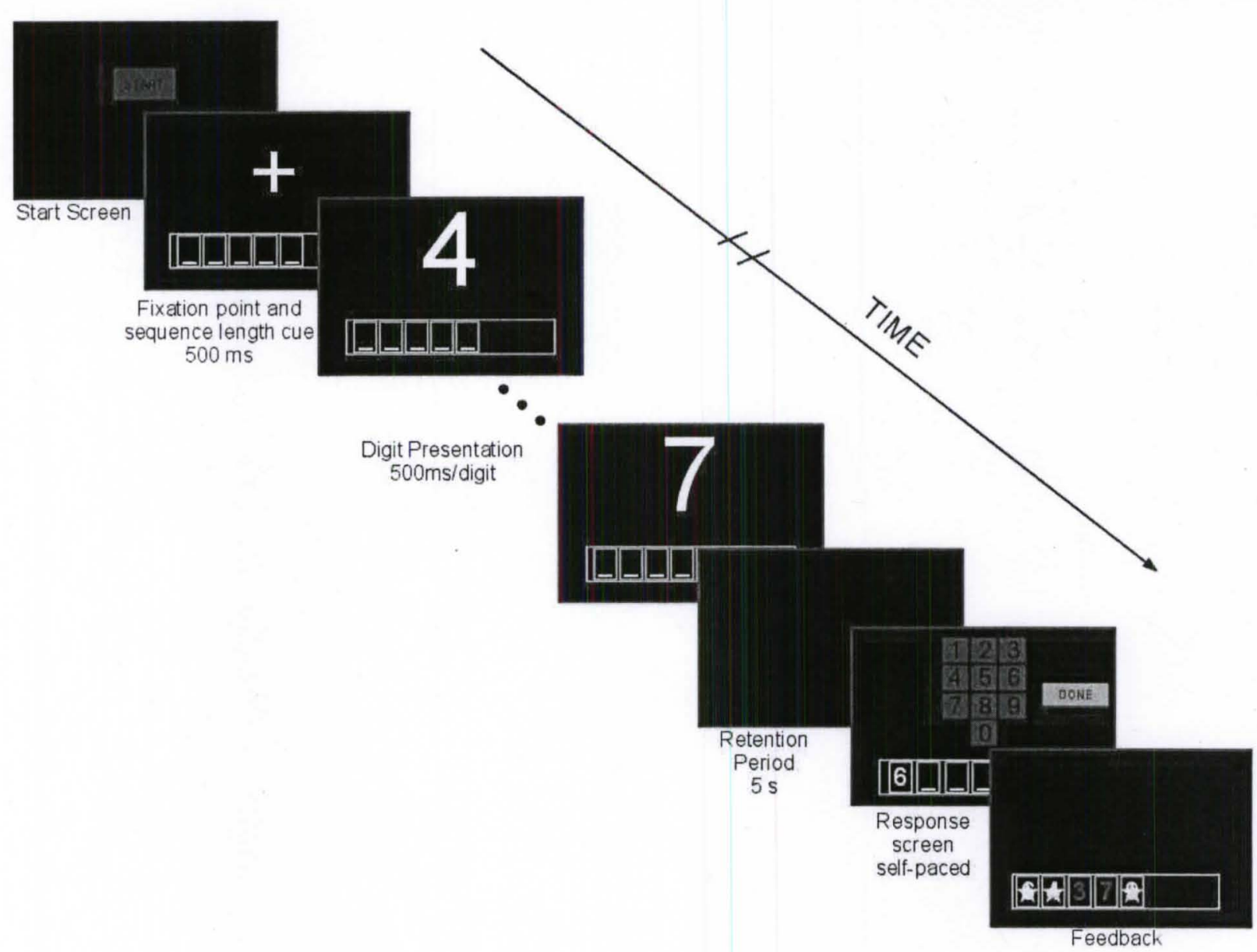

Figure 2.1. Schematic of the digit span task. Each trial was initiated with a $500 \mathrm{~ms}$ fixation cross at the center of the screen. Following the cue, a stream of digits was presented. After the retention interval, a response keypad appeared on the screen for participants to enter their responses. Participants then clicked on a green "done" button which initiated a feedback screen. Feedback consisted of presenting the entire list of digits that the participant had entered, with either happy faces or stars representing the items they had correctly named, and the digits incorrectly named in red 
Presentation of auditory materials. Following an initial practice run in quiet, the two distracter conditions and the quiet condition were blocked by run. Blocks were presented in random order. In order to elicit the greatest ISE possible, distracters were heard during presentation of the STM task, as well as during the $5 \mathrm{~s}$ retention interval. Distracters were not heard during the response phase. Participants completed eight runs in each distracter condition. This resulted in 35-40 data points at each sequence length for each individual. This is consistent with other studies which generally run 2025 trials at a single sequence length (Jones et al. 1993; Jones \& Macken, 1993). Runs lasted approximately ten minutes each. Participants were given the opportunity for breaks between runs. Participants generally were able to complete the study in three two-hour sessions.

\section{Results}

Effects of sequence length on task. Percent correct on the visual STM task was determined using methods typical of standardized tests of digit span (Dempster, 1981). If one or more digits in a sequence were recorded incorrectly, the entire trial was considered incorrect. Percent correct was thus determined by dividing the number of correct trials (trials without errors) by the total number of trials for each sequence length and multiplying this result by 100 .

Participants were tested over a range of sequence lengths. This range was determined by the individual performance of the participant, as described in the methods section. Examples of individual plots are shown in Figure 2.2. The purpose of this method was to test if the amount of ISE the participant experienced increased as a 
function of the difficulty of the task (difficulty was defined as an increase in sequence length). Span was defined as the maximum sequence length which produced performance closest to $50 \%$ correct, in quiet. Ranges of sequence lengths varied, with some participants tested across as many as eight sequence lengths, and some as few as four. To address this issue, the percentages of correct trials were extracted for each individual at three sequence lengths: Span, Span -1 and Span +1 . Figures 2.3 and 2.4 show these extracted data points for all participants. Figure 2.3 displays the child data, and Figure 2.4 displays the adult data. Mean percent correct for each of the sequence levels are shown. Three children and four adults showed nonmonotonic functions. Analyses were run excluding the data of these individuals, but the results were not affected. Thus, for the final analysis all individuals were included. Figure 2.5 displays a histogram showing the distribution of spans for all participants. Distributions for Span 1 and Span +1 have a similar shape, but are shifted either to the right $(\operatorname{Span}+1)$ or to the left (Span - 1) by one sequence length unit.

Data were entered into a $3 \times 3$ (Span Level [Span, Span - 1, Span +1] x Condition [quiet, letters, tones]) repeated measures ANOVA. Figure 2.6 shows the percentage of correct trials for each of the three chosen span levels across the three auditory conditions for all participants. Overall, performance decreased, as sequence length increased from Span - 1 to Span +1 . This effect is similar for each of the three different auditory distraction conditions (quiet, spoken letters, noise).The highest percent correct was at Span - 1 under the quiet condition, and the lowest percent correct was at Span +1 under the letters condition. This result was upheld by significant main effects of span level, $F(2,96)=37, p<.001$, as well as a significant main effect of condition, $F(2,96)=35.5, p$ 
$<.001$. No significant interaction was found between span level and condition, $F(4,192)$ $=.806, p=.522$. Given that the interaction was non-significant post hoc tests for condition were collapsed across span level. Tests using Bonferonni correction (.05/2) showed that the significant difference in auditory condition was between letters and quiet, $t(48)=-7.19, p<.001$, the speech condition had a significantly lower percentage correct. And the difference between the quiet and tones condition was non-significant, $t(48)=$ $2.61, p=.037$. This detail is important as other studies have shown that speech and nonspeech distracters would cause an equal amount of ISE (Jones \& Macken, 1993). Possible explanations for this difference will be considered in the discussion section.

Finally, a critical finding was the lack of interaction between the difficulty of the task, as measured by Span Level, and the auditory condition. A greater ISE was not seen under the most difficult condition (Span +1 ). Thus, an individual will not necessarily experience greater amounts of distraction from a speech distracter under those conditions which require more cognitive effort.

Age Group Differences. A secondary analysis was designed to test the hypothesis that children would show a greater level of disruption with speech distracters than adults. Because the results of the first analysis showed no increase in ISE as sequence length increased, the remaining analyses only used data taken at the participant's span. In order to test age differences, the percentage of correct trials on the visual STM task was entered into a $2 \times 3$ (Age [child, adult] $\times$ Condition [quiet, letters, tones]) repeated measures ANOVA. An alpha level of 0.05 was used. 

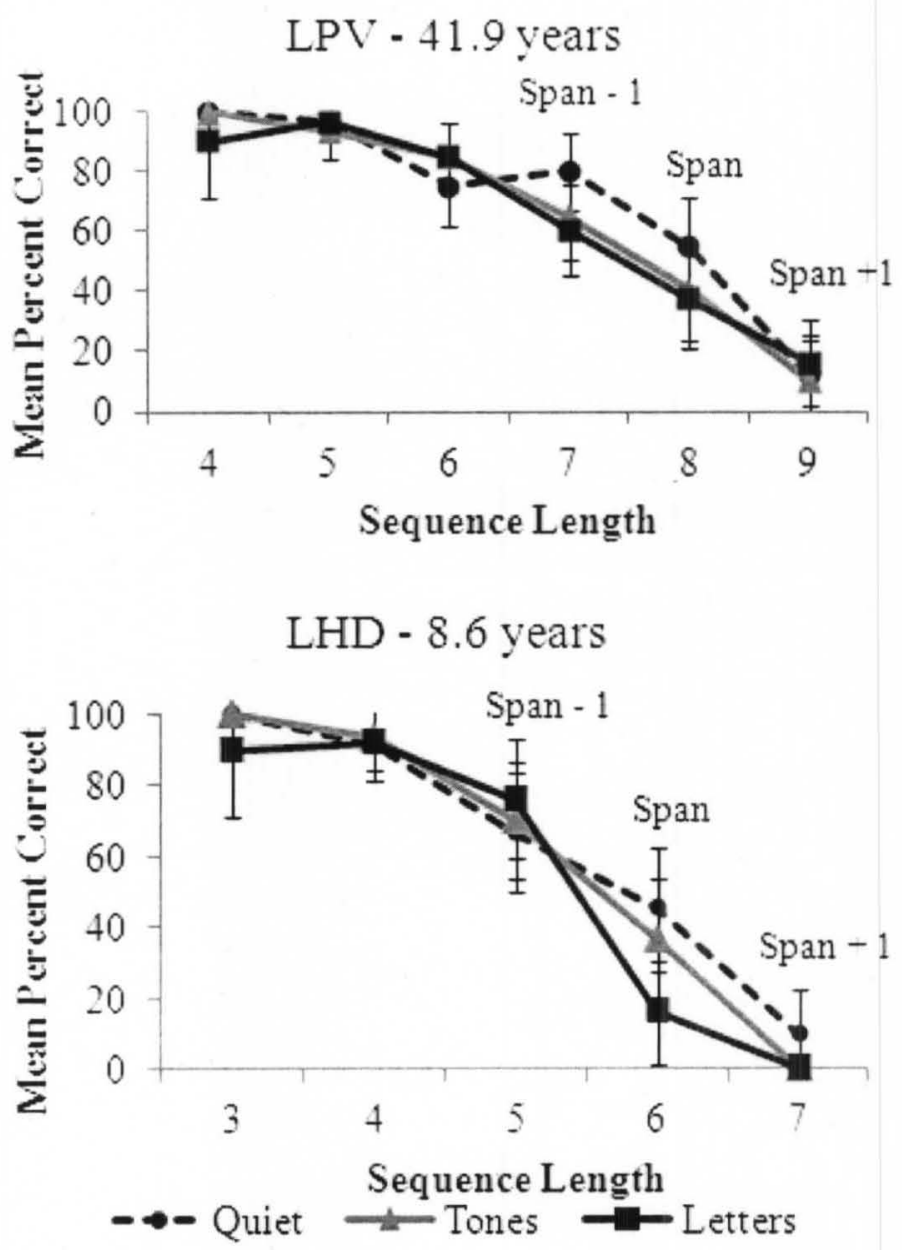

Figure 2.2. Results from the digit span test for two representative participants. Participant code and participant age in years is displayed at the top of each panel. The percentage of correctly reported digits in a sequence is plotted as a function of sequence length for each of the three distraction conditions: quite, tones, letters. Bars indicate $95 \%$ confidence intervals, which were calculated using the binomial variance. For each participant, the range of sequence lengths was chosen from approximately perfect to chance performance. Span was defined as the maximum sequence length which produced performance closest to $50 \%$ correct, in quiet. The ISE is indicated by reduced percentage correct for either the letters or tones condition. 


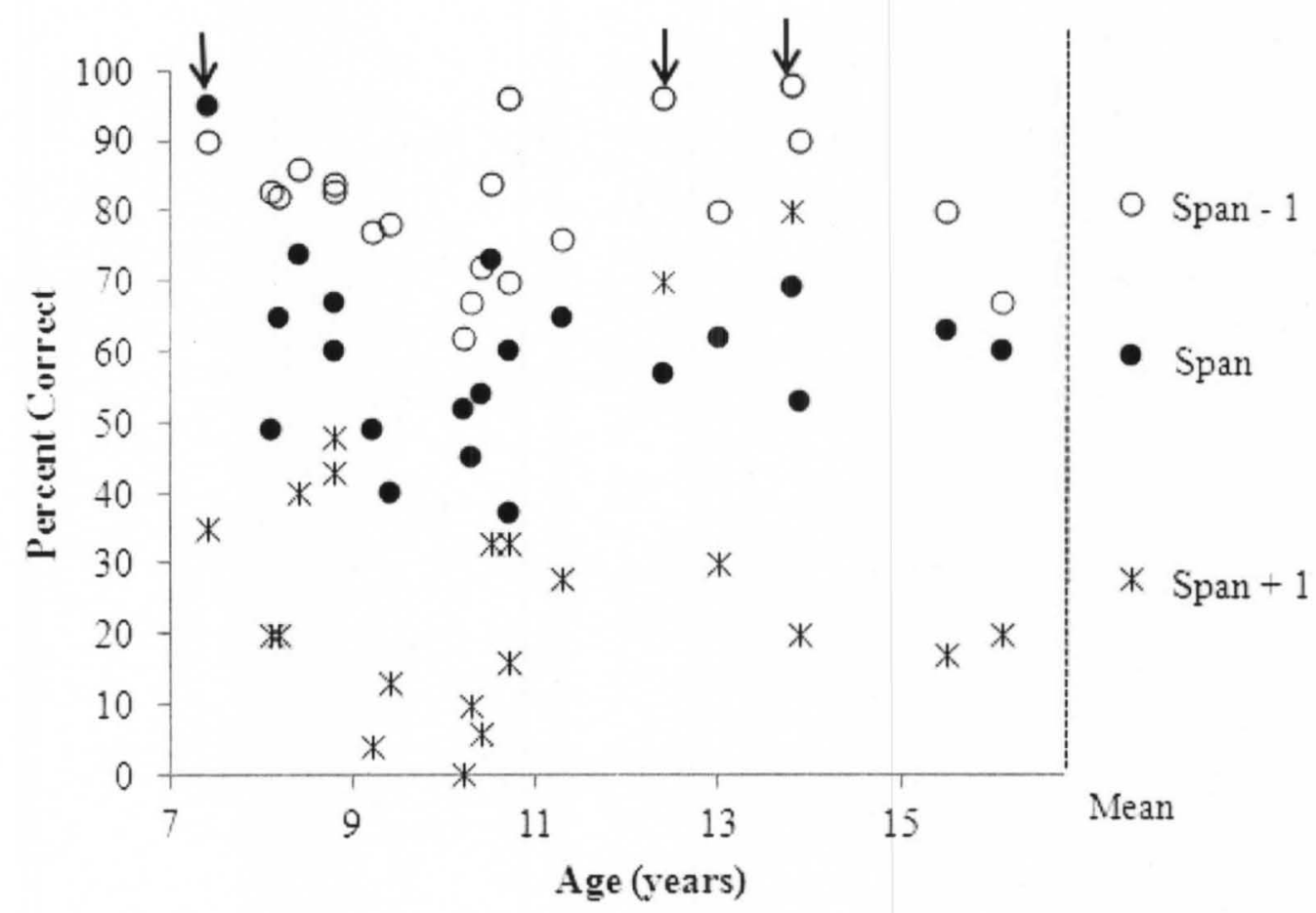

Figure 2.3. Span level data for child participants. Data shown are percent correct digit span performance in quiet. Three span levels are represented. Data points are ordered by age, from youngest to oldest. Group mean performance is displayed on the far right (Span $-1, M=81$, Span, $M=59.4$, Span $+1, M=27.9$ ). Three individuals showed nonmonotonic functions. These individuals are indicated with arrows. 


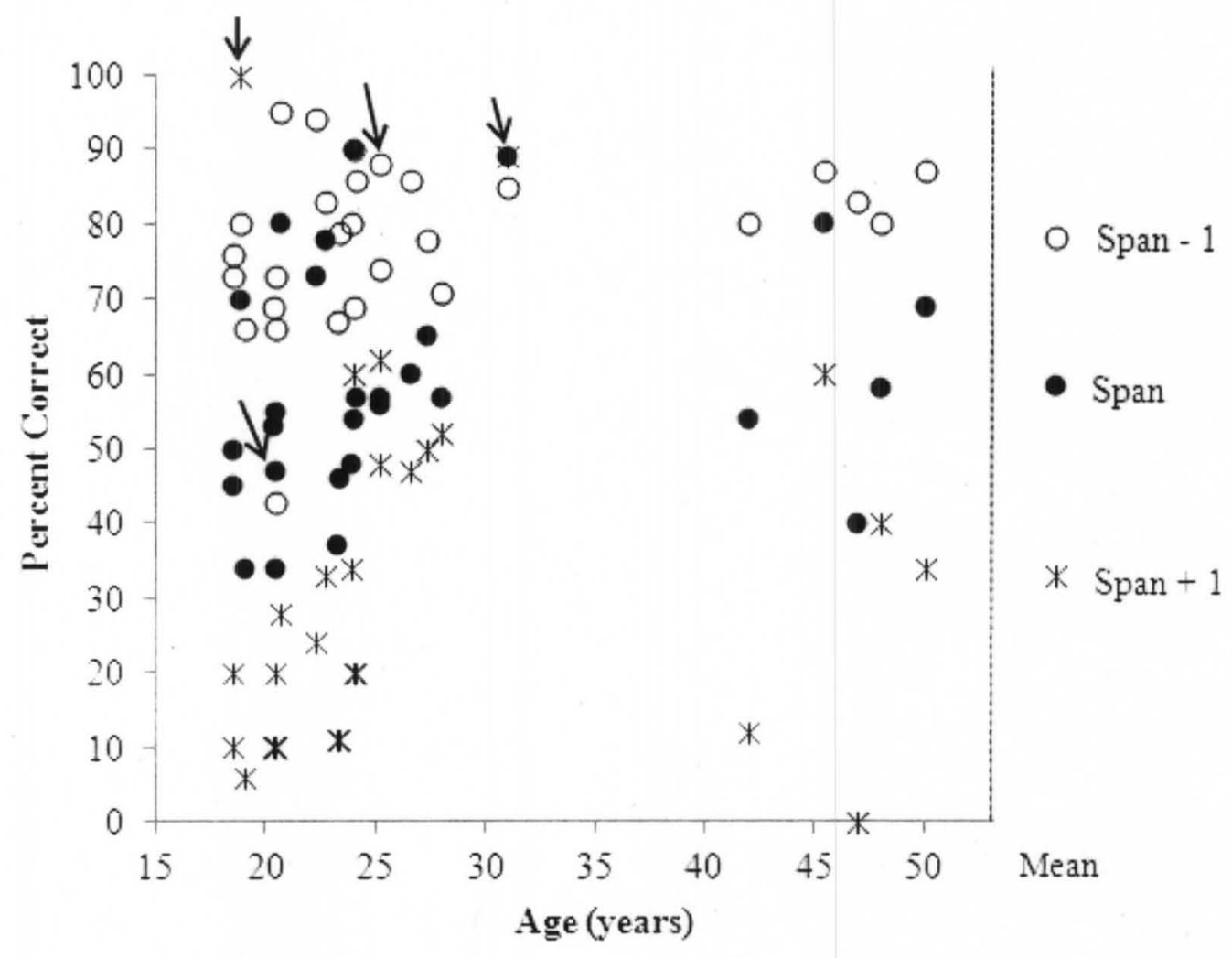

Figure 2.4. Span level data for adult participants. Data shown are percent correct digit span performance in quiet. Three span levels are represented. Data points are ordered by age, from youngest to oldest. Group mean performance is displayed on the far right (Span $-1, M=78.1$, Span, $M=58.4$, Span $+1, M=32.8$ ). Four individuals showed nonmonotonic functions. These individuals are indicated by arrows. 


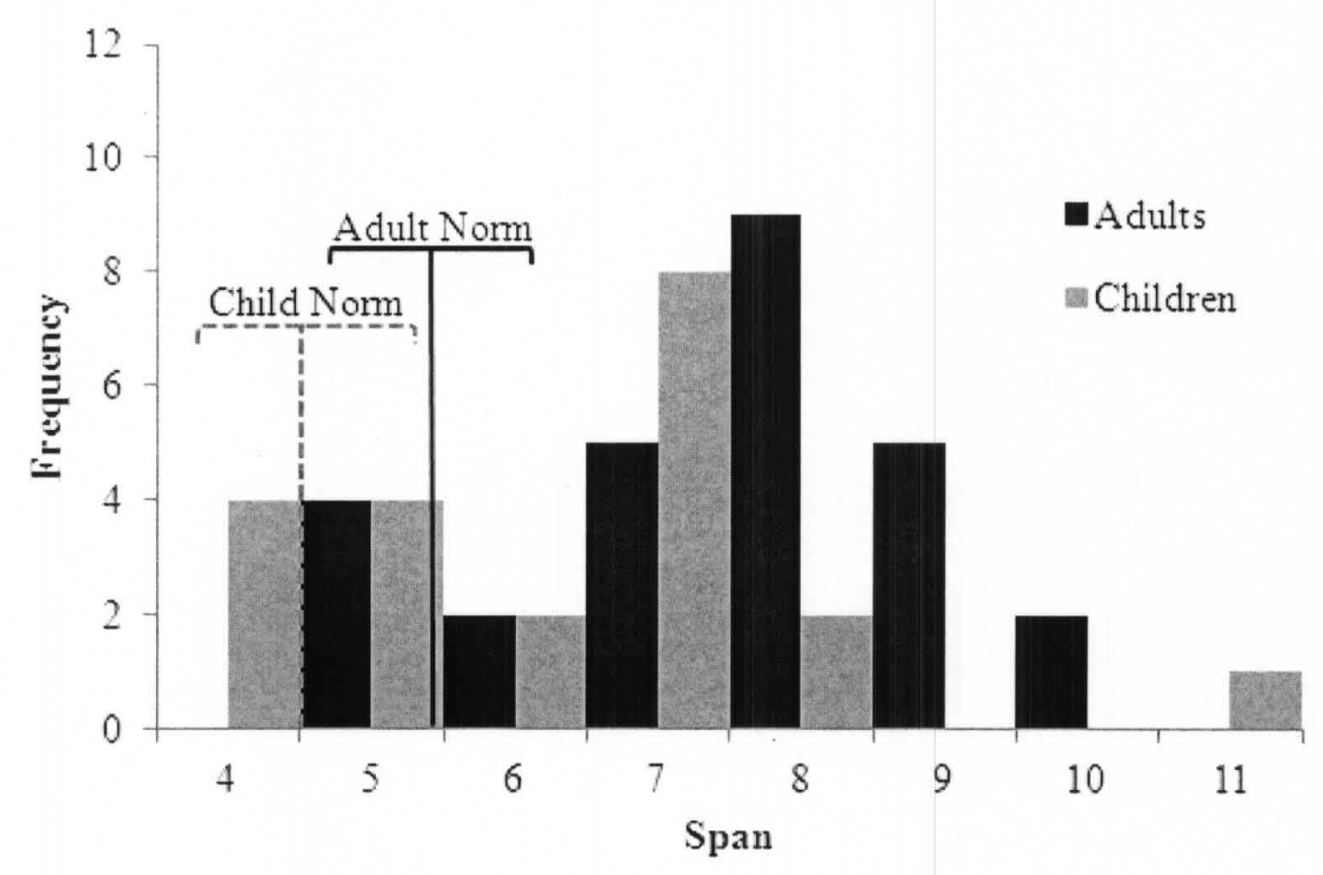

Figure 2.5. Histogram displaying the distribution of digit span in quiet among children $(M=6.2, \mathrm{SD}=1.7)$ and adults $(M=7.6, S D=1.5)$. Normative mean spans (Orsini et al., 1987) are indicated by the vertical lines. The gray dotted line is the mean span for children $(M=4.5, S D=.95)$. The black line represents mean span for adults $(M=5.4$, $S D=1.05)$. One standard deviation is represented by the brackets above. The sample of children and adults in this study had average spans that were higher than norms. 


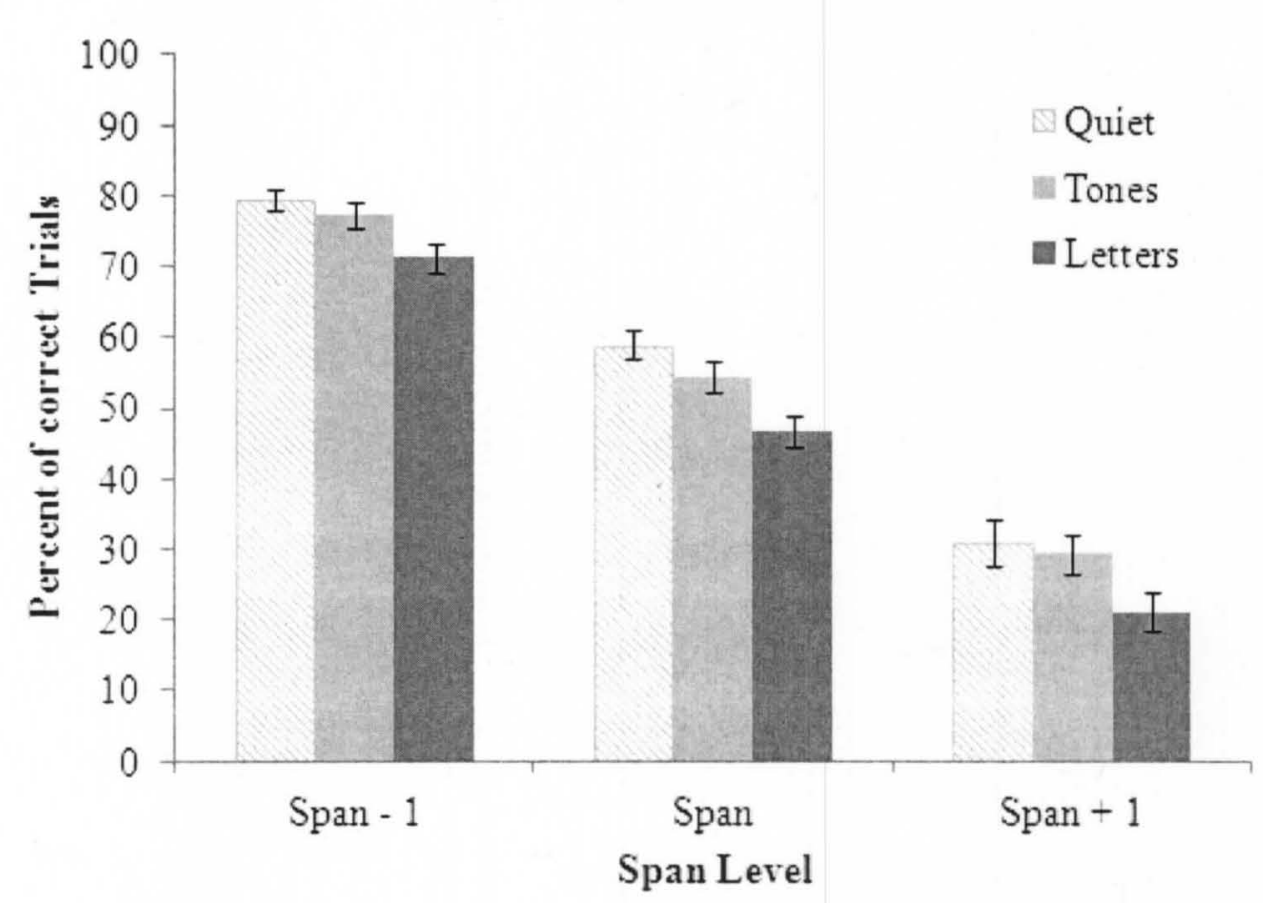

Figure 2.6. Results of serial recall task with auditory distraction. Group mean percentage of correct trials tested at each of three span levels for all participants $(n=50)$. Data show performance with each of the three auditory conditions. Performance is similar across the three span levels. Error bars represent one standard error of the mean. 
Figure 2.7 illustrates the levels of percent correct in each of the conditions, for the two age groups. When span is controlled for it is apparent that the age groups perform similarly. The ANOVA showed only a significant main effect of condition $F(2,94)=26.27, p<.001$. The main effect of age group was non-significant $F(1,47)=$ $.342, p=.561$, as well as the interaction between age and condition, $F(2,94)=.462, p=$ .632. The main effect of condition demonstrated that all individuals experienced an ISE, however, post hoc comparisons using Bonferroni correction $(.05 / 4)$ showed that while there was a significant difference between the letters and quiet conditions for the adults, $t$ $(27)=5.582, p<.001$ and for the children, $t(20)=3.19, p=.005$, no significant difference between the tones and quiet conditions was observed for either the adults, $t(27)=2.66, p=.014$, or the children, $t(20)=.991, p=.333$. This again demonstrates that non-speech distracters do not result in an ISE, even for children. The result of interest in this analysis however, was the lack of interaction between age group and condition. This result demonstrated no relationship between age and type of auditory materials. Thus, for this sample of participants, it may be concluded that children and adults were equally impaired by irrelevant speech.

Span group differences. The final question posed at the outset of this study asked whether individuals with shorter spans would be more affected by distracting sounds than individuals with longer spans. In past studies of the ISE in adults, the ISE was measured by examining number of errors across serial position of the sequence of digits to be remembered. A serial position analysis allows an examination of errors at each point along the sequence. For example, an individual may always get the first and 


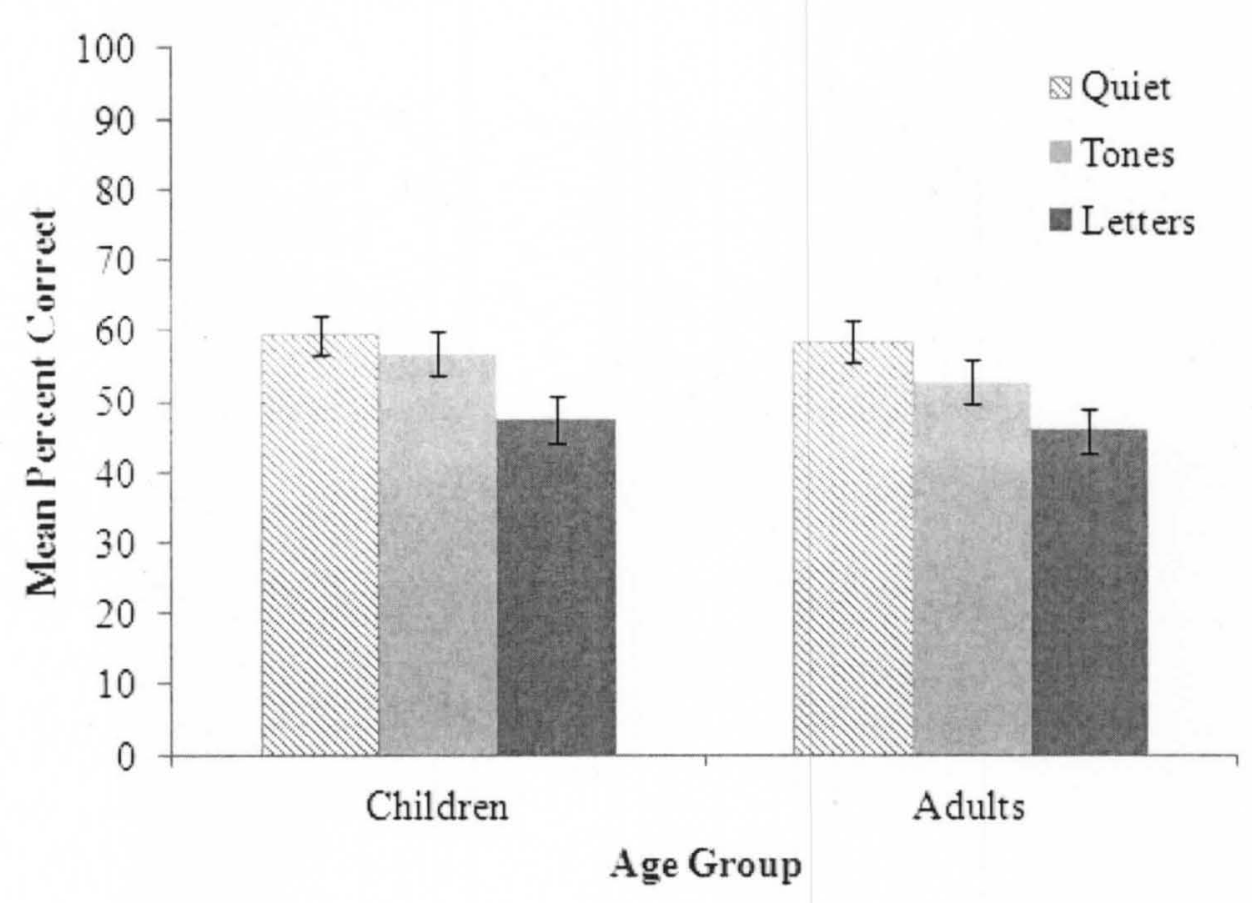

Figure 2.7. Results of the serial recall task by age group. Shown are age group mean percentages of correct trials on a digit serial recall task at span only. Performance in each of the three auditory conditions is represented. Error bars represent one standard error of the mean 
last digits in a sequence correct, but under conditions of auditory distraction, their errors on the second-to-last digits could increase. Typically, during previous studies all individuals were tested at the same sequence length. This allowed for a serial position analysis using group data. However, information about individual differences in performance attributed to span is lost. Large amounts of individual variability in the ISE have been found in other studies (Ellermeier, \& Zimmer, 1997), but no explanation has been offered for these differences. A serial position analysis which examines different groups at different spans has not been reported in previous studies. This type of analysis may help to pinpoint a source for these individual differences.

In order to compare the present data with past studies which have examined the effects at only one sequence length, the effects of auditory condition across serial position were analyzed. However, rather than comparing all individuals on only one sequence length, participants were divided into groups based on their memory span. These groups were determined based on collected norms; however the spans of the children and adults of this sample were skewed overall towards higher than normal spans. The child groups were formed by referencing the reported norms of mean digit span for children. These norms report a mean digit span of 4.5 digits $(S D=.95)$ for children (Orsini et al., 1987). Thus, the children were divided into two span groups: A normal span group and a high span group. The normal span group $(n=10)$ contained individuals with a range of spans between 4-6 digits. Individuals whose spans were two standard deviations above the mean of reported norms were placed into a high span group $(n=11)$ with a range of spans between 7-11 digits. The adults were also divided using reported norms. Orsini et al (1987) found a mean digit span of 5.4 digits $(S D=$ 
1.05 ) for adults. Adults were initially divided into two groups based on these reported norms as well. Individuals with spans at the mean or within two standard deviations were placed into one group $(n=10)$, and the remaining individuals into a high span group. However there were a far greater number of individuals in the high span group. Therefore, rather than divide the adults into only two groups, three groups were formed; normal span $(n=10)$ with a span range of $5-7$, middle span $(n=11)$ with a span of 8 , and high span $(n=7)$ with a span range of 9-10. See Figure 2.8 for illustration of distribution of span group by age. The sequence lengths to be analyzed for each group were chosen based on the group mean spans. This entire procedure facilitated a span group analysis of serial position errors.

The dependent variable analyzed was the percent of errors at each serial position. Percent of errors was then compared for each of the three auditory conditions. Five separate $n \times 3$ repeated measure ANOVAs were used to analyze within group differences on the dependent variable of the percent of errors on the visual STM task. The levels of the first factor were $n$ serial positions (number of serial positions were determined by group). The levels of the second factor were the three auditory conditions: quiet, letters and tones. An alpha level 0.05 was used.

Serial position curves for the two child groups are shown in Figure 2.9, and serial position curves for the three adult groups are shown in Figure 2.10. The normal span child and normal span adult serial position curves show a shape that is typical of past studies of the ISE. There are clear primacy and recency effects (the first and last serial positions have the least number of errors), and the effect of the letters distracter is clear 


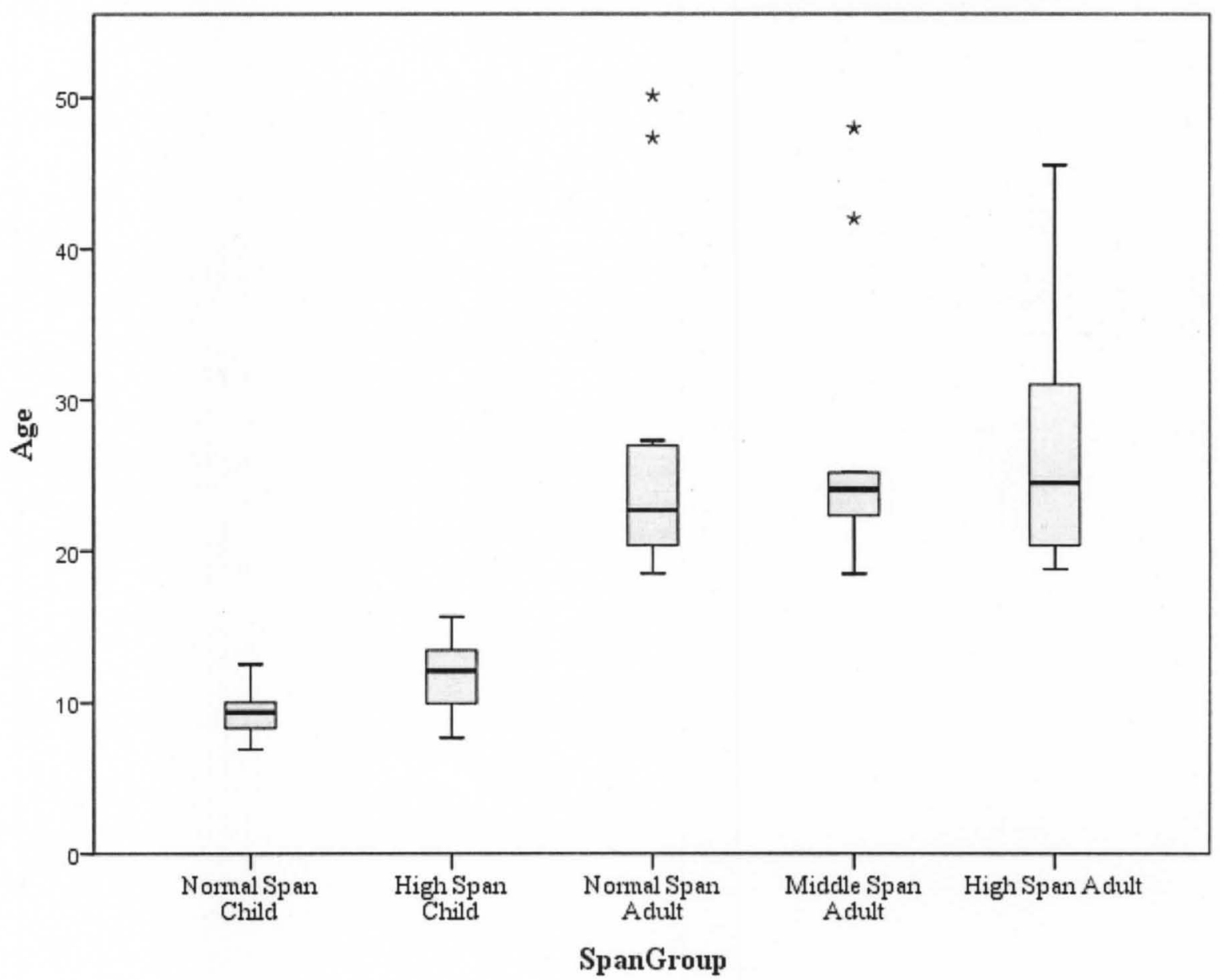

Figure 2.8. Box plot shows distributions of age for each of the five span groups by age. Cohorts are (a) normal span child group (spans of 3-5) (b) high span child group (spans of 6 and greater) (c) normal span adult group (spans of 4-7) (d) middle span adult group (spans of 8 ) and (e) high span adult group (spans of 9 or more). Means are represented by the horizontal bars intersecting the boxes. Whiskers represent the highest and lowest values that are not outliers or extreme values. Outliers (values that are between 1.5 and 3 times the interquartile range) are indicated by stars. 


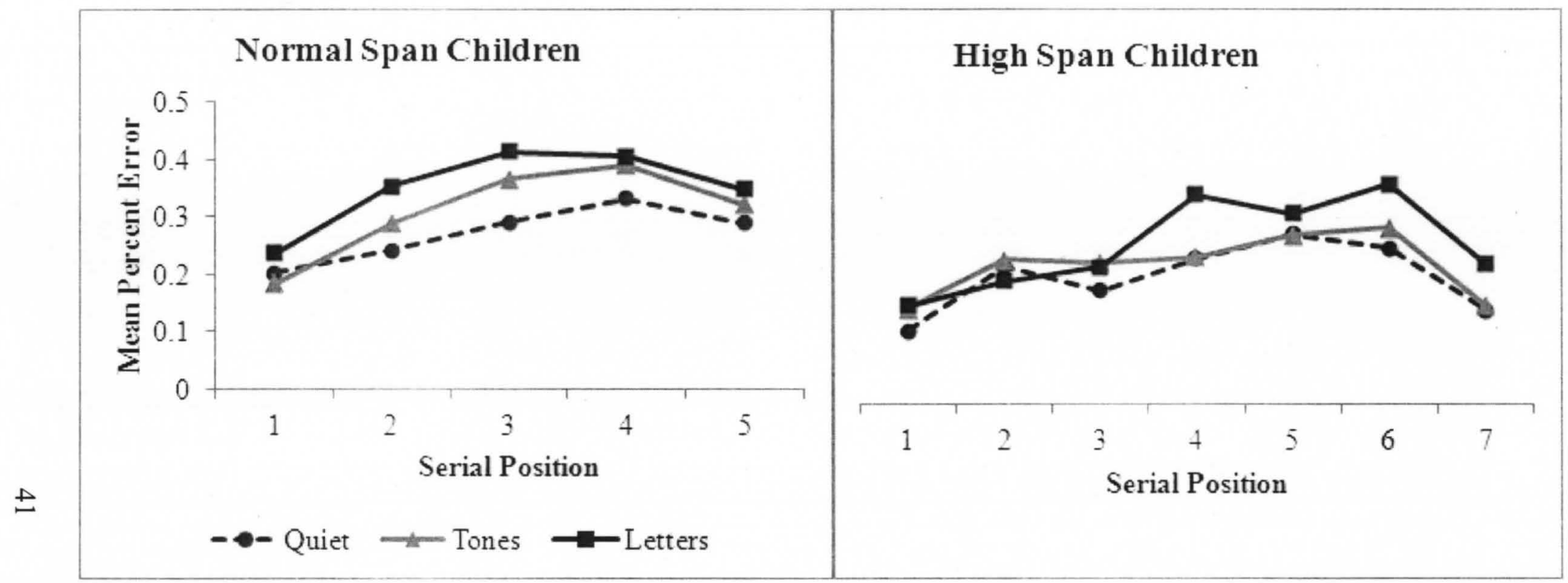

Figure 2.9. Data show mean percent error for normal span child group $(n=10)$ tested at a sequence length of 5 , as well as data for the high span child group $(n=11)$ tested at a sequence length of 7 . Normal span children were significantly more affected by the letters distracter than were the high span children as indicated by the increase in errors across sequence length. 


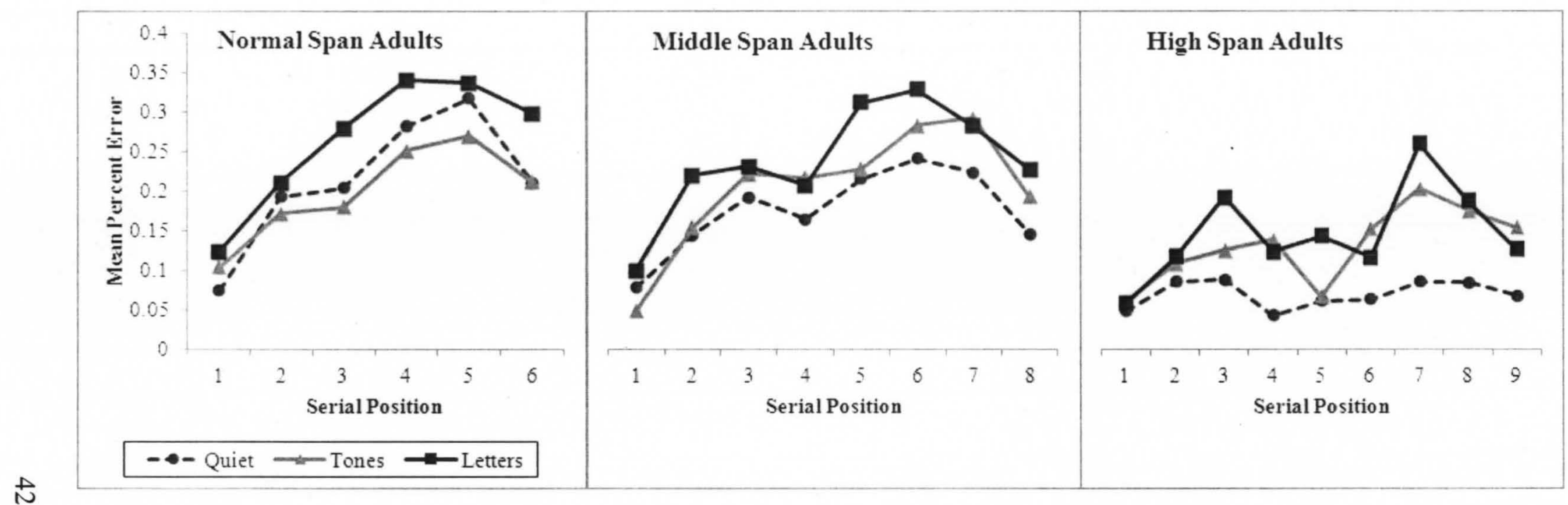

Figure 2.10. Data show mean percent error for normal span adult group $(n=10)$ tested at a sequence length of 6 , data for the middle span adult group $(n=11)$ tested at a sequence length of 8 , and data for the high span adult group $(n=7)$ tested at a sequence length of 9. Normal and middle span adults were significantly more affected by the letters distracter than were the high span adults as indicated by the increase in errors across sequence length 
Table 2.1.

Summary of Separate Span-Group ANOVAs

\begin{tabular}{|c|c|c|c|c|c|c|c|c|c|c|}
\hline \multirow[t]{2}{*}{ Group } & \multirow{2}{*}{$\begin{array}{l}\text { Sequence } \\
\text { Length } \\
\text { Analyzed }\end{array}$} & \multicolumn{3}{|c|}{ Effect of Serial Position } & \multicolumn{3}{|c|}{ Effect of Condition } & \multicolumn{3}{|c|}{ Interaction } \\
\hline & & $d f$ & $F$ & $\bar{p}$ & $d f$ & $\bar{F}$ & $p$ & $d f$ & $\bar{F}$ & $\bar{p}$ \\
\hline \multicolumn{11}{|c|}{ Children } \\
\hline $\begin{array}{l}\text { Normal span } \\
\qquad(n=10)\end{array}$ & 5 & 4,36 & 9.93 & $<.001$ & 2,18 & 3.94 & .038 & 8,72 & 1.38 & .218 \\
\hline $\begin{array}{c}\text { High Span } \\
\quad(n=11)\end{array}$ & 7 & 6,66 & 10.06 & $<.001$ & 2,22 & 1.64 & .220 & 12,132 & 2.61 & .004 \\
\hline \multicolumn{11}{|c|}{ Adults } \\
\hline $\begin{array}{l}\text { Normal span } \\
\qquad(n=10)\end{array}$ & 6 & 5,45 & 9.27 & $<.001$ & 2,18 & 5.46 & .014 & 10,90 & 1.22 & .288 \\
\hline $\begin{array}{l}\text { Middle Span } \\
\quad(n=11)\end{array}$ & 8 & 7,63 & 11.74 & $<.001$ & 2,18 & 3.96 & .037 & 14,126 & 1.01 & .451 \\
\hline $\begin{array}{l}\text { High Span } \\
\quad(n=7)\end{array}$ & 9 & 8,48 & 3.38 & .004 & 2,12 & 2.38 & .135 & 16,96 & .719 & .768 \\
\hline
\end{tabular}


across all serial positions. The middle span adult group also shows a similar effect of condition, but rather than a primacy and recency effect, there seems to be a "chunking" (Miller, 1956) of the first four and last four digits. The high span child and high span adult groups show a somewhat atypical shape. The serial position curve seems to be flattened, and the effect of recency and primacy is no longer as pronounced. The effect of condition is also somewhat less apparent, and appears to depend on serial position. Table 2.1 displays the results of the five span group ANOVAs. For the normal span child group as well as the normal and middle span adult groups there was a significant effect of both serial position and condition and a significant interaction between the two. For the high span children and the high span adult group there was only a significant effect of serial position, and no effect of condition. These results indicate that those individuals with spans above the norm for their age group are less affected by the presence of irrelevant noise than those individuals with average spans.

\section{Discussion}

The three primary goals of this experiment were to (1) investigate the ISE while controlling for differences on the level of performance in the serial recall task, (2) to examine developmental differences in susceptibility to the ISE, and (3) to investigate the relationship between individual memory capacity and susceptibility to the ISE. Regarding the first goal, it was found that if individuals are tested at various sequence lengths, from undemanding to difficult, the effect of condition does not change in a meaningful way. Thus, the individual variability in the susceptibility to an irrelevant distracter is not directly related to the sequence length of the visual task. A secondary 
goal was to investigate whether children and adults are equally affected by a speech distracter. A previous study found that young children had greater difficulty ignoring speech than tones (Elliot, 2002). Elliot used Cowan's theory of attentional recruitment (2008) to explain these findings. She posits that children have fewer attentional resources and that speech requires greater resources than tones. Thus, speech would recruit more of the resources away from the visual task. Under this explanation it is not clear why adults would not show the same difference between speech and tones.

The spans of this sample were substantially higher than the norms. It is unlikely however that this was due to methodological differences between the current study and the normative study (Orsini et al., 1987). Although the methods employed in this experiment to determine span were not typical, they were designed to mimic the outcome of standardized digit span tests as closely as possible. A standardized digit span test will find as the individual's span the longest sequence length which they can recall $50 \%$ of the time. For the current experiment, span was defined as the longest sequence length in which the individual was operating at a performance closest to $50 \%$ correct. It seems much more likely that sampling considerations may have influenced the range of observed spans. Orsini et al. (1987) have also shown that STM span is related to education level. There is also evidence that STM span in children is related to parental schooling (Hoffert, Smith, McLoyd, \& Finkelstein, 2000; Wood, 2002). Participants in our experiments, including the children, are generally recruited from a university population. There is a possibility that the education level of the sample was higher than the sample used by Orsini et al. 
Data from Experiment 1 show that the differential disruption of speech is not dependent on the age of the listener. These results are in-keeping with the concept that speech may require greater resources, but it may be more accurate to use STM capacity as a measure of these resources rather than age. The serial position analysis shows that the greater effect of a speech distracter is more closely related to the individual's STM capacity than their age. Individuals with lower capacities are more affected by irrelevant competition from speech. In most previous studies of the ISE the analysis used is one of a comparison of serial position errors. When the current data were analyzed using a similar method a clear pattern did emerge. A comparison of errors at varying sequence lengths revealed that the amount of distraction experienced was not because of the difficulty of the serial position task. However the ISE does in fact seem to be related to an individual's STM capacity. There was a significant effect of auditory condition found in the normal span groups, but not in the high span groups, although the pattern of the effect across serial position for the high span group appears to be complex. Thus, when level of performance is controlled for and group data is analyzed using a meaningful sequence length as a reference, the ISE is greater in those individuals with a low to average STM capacity.

According to the Working Memory Model posed by Baddeley, the ISE results from the disruption of the phonological loop (Baddeley, 1982). The current findings agree with this theory if one conceptualizes STM capacity as a measure of the efficiency of the phonological loop. These results could also potentially explain why there has been a difficulty in demonstrating a relationship between WMC and the ISE (Beaman, 2004; Elliot \& Cowan, 2005). Measures of working memory such as the OSPAN may better 
gauge an individual's ability to shift attention. Rather than pointing to a difference in the ability to task switch to explain the ISE, the differences may lie with the rehearsal strategies of the individual. That is, individuals with a smaller STM capacity may be more challenged by subvocal rehearsal, which would recruit attention away from the central executive leaving them more easily disrupted by speech distracters.

There has been an assumption in past studies of the ISE that those participants who are working at their individual span are all operating at the same level of performance. This may not be the case for individuals with unusually large spans. In a review examining sources of individual variability of STM capacity, Dempster (1981) reported that non-strategic variables such as susceptibility to interference correlate most strongly with capacity, even when age is partialled out (Dempster, 1981) Accordingly, the best explanation for the individual variability in susceptibility to the ISE found in the present study may be these non-strategic variables. 


\section{CHAPTER III}

\section{EXPERIMENT 2}

Results from Experiment 1 support Baddeley's phonological loop model rather than the changing state theory--auditory sounds that do not contain speech will not disrupt the serial recall of verbal material. Results from the serial position analysis in Experiment 1 indicated two further significant findings with regards to these competing theories. (1) Individuals with a greater STM capacity show less susceptibility to irrelevant sounds, regardless of content. In addition, those individuals with a smaller capacity show a difference between speech and non-speech auditory stimuli, with speech distracters causing greater amounts of distraction. (2) These differences did not seem to be age-related, i.e. high span children showed the same advantages as high span adults, and normal span children showed the same decrement in performance as normal span adults.

In addition to making predictions about the content of the auditory distracter, the Working Memory Model also makes predictions regarding the nature of the STM task. According to this model, visuo-spatial materials are encoded using the visuo-spatial scratch-pad. Since auditory materials do not gain access to the scratch-pad, memory performance on visuo-spatial tasks should not be degraded by auditory materials. First, Experiment 2 a employed an articulatory suppression task to demonstrate that the visuo- 
spatial STM task to be used required no verbal rehearsal. Then, in order to further test the predictions of the Working Memory Model, Experiment $2 \mathrm{~b}$ investigated the ISE using this nonverbal visuo-spatial STM task.

Past research shows that potential sources of individual differences in STM capacity may relate to rehearsal strategies. Experiment 2 investigated the role of rehearsal strategies by examining the effects of irrelevant sounds on a nonverbal STM test. If the source of the ISE is the phonological loop, as Baddeley's Working Memory Model predicts, and capacity is determined by the efficiency of verbal rehearsal strategies, a STM task which requires no verbal rehearsal would show no effect of irrelevant speech for either high span or normal span individuals.

Developmental differences in the ISE can be tested using the same paradigm described above. Children's memory capacities are smaller than adults. It has been hypothesized that this difference in capacity is attributable to rehearsal strategies, and not necessarily differences in attention (Dempster, 1981). These two factors that may predict individual differences in memory capacity, rehearsal strategy or attention, could also play different roles in developmental differences of the ISE. If the developmental difference in capacity is determined by attentional resources and not by rehearsal strategies, children should still demonstrate an ISE even with nonverbal materials.

\section{Experiment 2a}

A group of participants were tested on both a digit task and a visuo-spatial task using a technique known as articulatory suppression (AS). This technique requires the participant to utter some irrelevant sound such as "the" during a STM task. It has been 
shown to disrupt encoding of the visual materials. This disruption occurs because the participant is unable to use subvocal rehearsal to commit the to-be-remembered stimuli to memory (Baddeley, Lewis, \& Valar, 1984). The visuo-spatial task created for this study was designed to require no verbal rehearsal. If this design was successful, then a concurrent AS task performed during the visuo-spatial task would not disrupt recall.

\section{Method}

Participants. Forty-two participants were recruited using either an internet news source provided by the University of Louisville, flyers, or through personal contacts. There were 24 adults ( 15 female), ages ranged from 18.0 to 41.1 years $(M=$ $24.1, S D=6.0)$, and 18 children ( 11 female) ages ranged from 7.1 to 12.8 years $(M=$ 9.9, $S D=2.1$ ). All individuals were screened for normal hearing by passing a $20 \mathrm{~dB}$ $\mathrm{HL}$ audiometric screening at octave frequencies $250-8000 \mathrm{~Hz}$. Participants were also screened for normal or corrected-to-normal visual acuity using a Snellen letter chart. Participants were asked to report any diagnoses of attention problems. Participants were compensated $\$ 10$ per hour. All testing was approved by the University of Louisville Internal Review Board.

Apparatus/materials. Participants were tested one at a time in a double-walled sound isolation chamber (Acoustic Systems). Software for the visual portion of the test was created using MATLAB.

Visual digit stimuli. Parameters of the digits portion of the test are identical to those reported in Experiment 1. 
Visuo-spatial Stimuli. Screen resolution was 1680x1050 pixels. A series of small white squares were presented in random locations on a black $7 \times 7$ inch panel located in the center of the screen. Squares were $50 \times 50$ pixels, and no square had a location less than 120 pixels from the previous square. Participants were seated approximately two feet from the computer monitor and this distance subtended a visual angle from the participant to the stimuli of approximately $1^{\circ}$.

Procedure. Digit Span test. Prior to testing, all participants were required to complete a digit span test. The methods of this test were based on standardized digit span tests. Each participant was required to memorize a sequence of digits. Each sequence length was presented twice. Sequences increased in length until the participant could no longer repeat the sequences accurately. Digit span was defined as the highest list length of digits at which the participant could reproduce at least one sequence length correctly.

Digits test with articulatory suppression. Participants were asked to repeat the word "the" at the rate of one repetition per second during the completion of a single run in quiet, at their predetermined span. A single run consisted of 20 trials of the digits test. They were asked to begin the repetition at the time that the digits appeared and throughout the retention interval. In order to ensure compliance with these instructions, the experimenter monitored the participant using a microphone placed in the booth. Participants were asked to discontinue the repetition when the response screen appeared.

Visuo-spatial span test. Each individual received a visuo-spatial STM span test. Prior to the beginning of each trial, the participant was presented with a cue, which 
informed them of how many squares they were about to see. Then, each participant was shown a number of squares one at a time at random locations on the screen. They were required to remember the location of the squares in the order that they saw them. Squares were presented for $500 \mathrm{~ms}$ with a $500 \mathrm{~ms}$ pause interval between. Following the presentation of the squares was a $5 \mathrm{~s}$ rehearsal period, after which all of the squares reappeared on the screen. The participants would then click on the squares in the order that they saw them. The participants were presented a range of sequence lengths beginning with two squares and increasing to ten. Each sequence length was presented twice. Visuo-spatial span was defined as the highest list length of squares at which the participant could reproduce at least one sequence length correctly. This method was based on a standardized method for determining visual- spatial STM span (Berch, Krikorian, \& Huha, 1998). See Figure 3.1 for a schematic of the visuo-spatial serial recall task

Visuo-spatial test with articulatory suppression. The stimuli were identical to those listed above. Participants were asked to repeat the word "the" at the rate of one repetition per second during the completion of a single run in quiet, at their predetermined span. They were asked to begin the repetition at the time that the squares appear and throughout the rehearsal period. In order to ensure compliance with these instructions, the experimenter monitored the participant using a microphone placed in the booth. Particiapants were asked to discontinue the repetition when the response screen appears. 


\section{Results}

In order to demonstrate that the visuo-spatial task required minimal verbal rehearsal on the part of the participant, data for an AS task were analyzed for each of the two memory tasks, digit span and visuo-spatial span. Within each group, paired sample $t$ tests were performed to test whether there was a significant increase in errors under conditions of AS. If the visuo-spatial task does not require verbal rehearsal, an individual should show no decrement in performance under this condition. Figure 3.2 illustrates the difference in performance across the two tasks. A significant difference between percent of correct trials on the digits task in quiet and the digits task under AS was found for the adults, $t(24)=-13.7, p<.001$, and children, $t(17)=-10.4, p<.001$. Performance of both the adults and children was significantly poorer in the AS condition. For the visuospatial task, adults showed no significant difference between the task in quiet and the task under AS, $t(24)=-.51, p=.618$. The children showed a slight but significant improvement in accuracy under the AS condition, $t(17)=2.91, p=.010$, although the reason for this is unknown.

These data show that AS reduces memory retention for the digit span task, but shows no significant effect on performance for the visuo-spatial task for adults, and possibly even enhances performance for children. These data support the prediction made by the Working Memory Model that a visuo-spatial task requires minimal active verbal rehearsal on the part of the participant. 


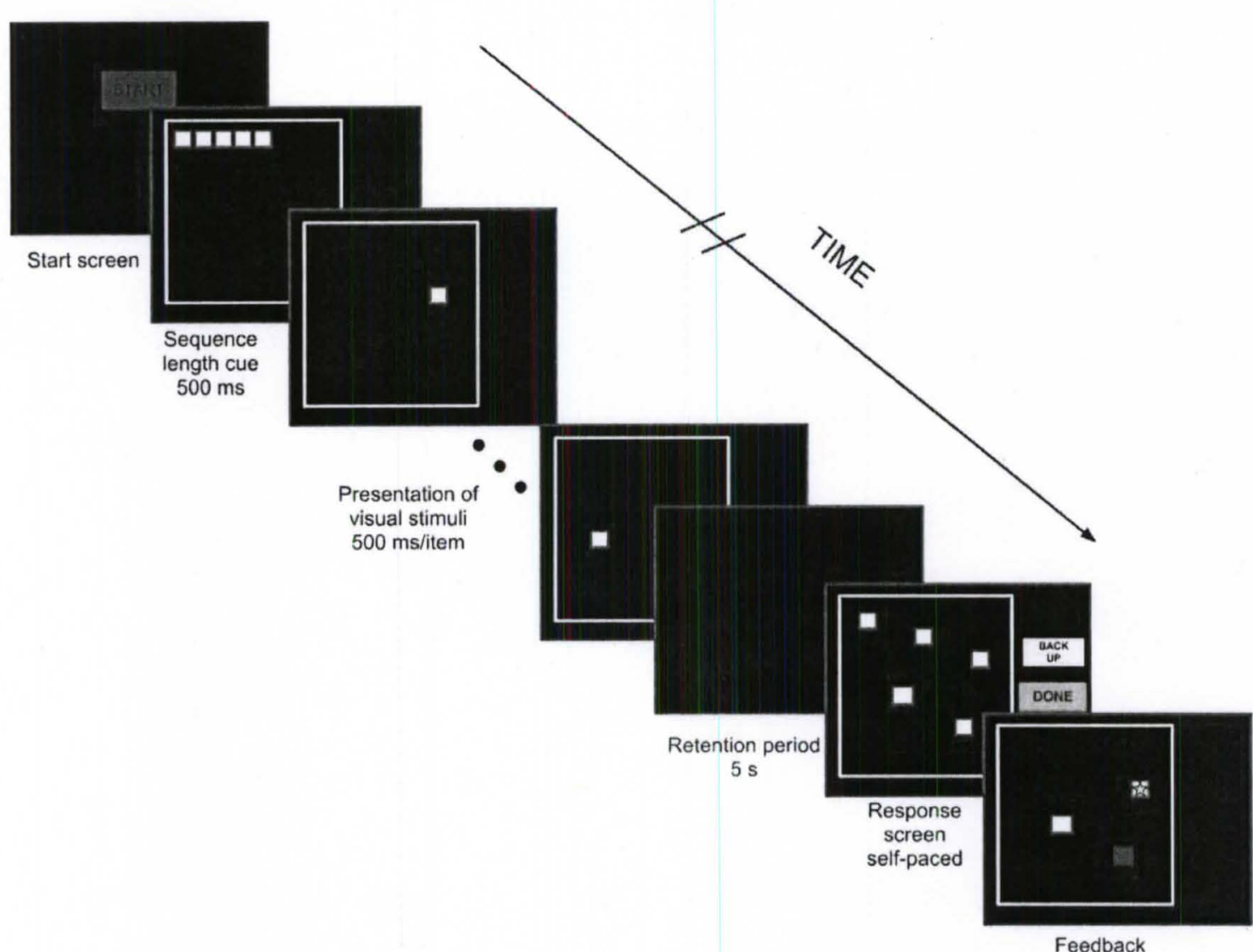

Figure 3.1. Schematic of the visuo-spatial serial recall task. Participants were shown a number of squares one at a time at random locations on the screen. They were required to remember the location of the squares in the order that they saw them. Following the $5 \mathrm{~s}$ rehearsal period, all of the squares re-appeared on the screen. The participants then clicked on the squares in the order that they saw them. Visuo-spatial span was defined as the highest list length of squares at which the participant could reproduce at least one sequence length correctly. 


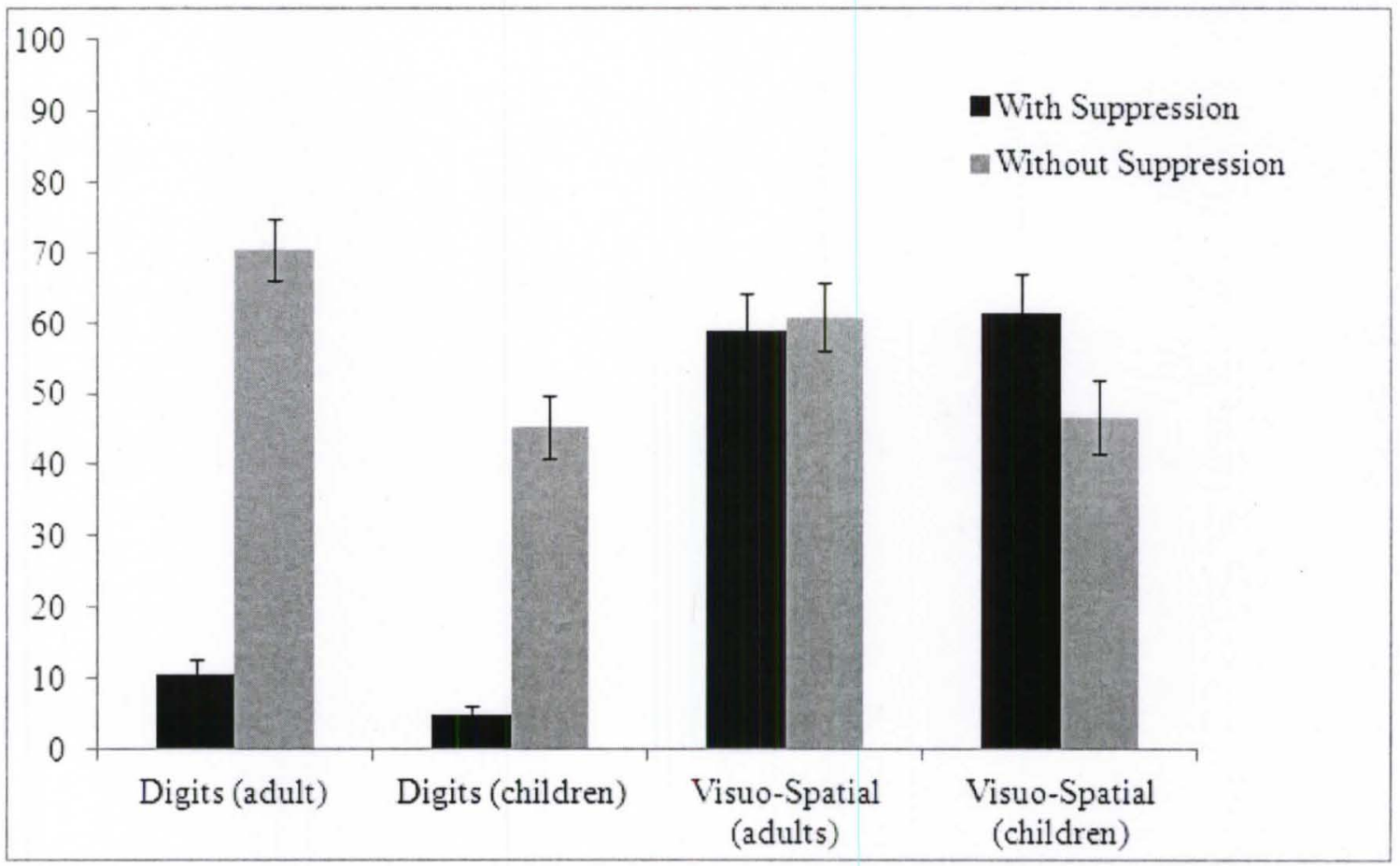

Figure 3.2. Group mean performances on the two memory tasks with either AS or no AS. Performance on the digits task with AS was significantly poorer than on a visuospatial task with AS. Children and adults showed similar effects of AS. Error bars represent one standard error of the mean. 


\section{Experiment 2b}

\section{Method}

Participants. Fifty participants were recruited using either an internet news source provided by the University of Louisville, flyers, or through personal contacts. There were 25 adults ( 14 female) and 25 children ( 15 female) recruited. Ages of children were restricted to an age range $(7.1-12.8, M=9.6$ years, $S D=2.0)$ which shows a large amount of developmental change in memory capacity (Orsini et al., 1987). The upper cutoff of the age range of the children is based on evidence that children begin to show adult-like capacities by the age of 12 (Dempster, 1981). Adult age range was $18.0-41.1$ years $(M=24.1$ years, $S D=6.0)$. All individuals were screened for normal hearing by passing a $20 \mathrm{~dB} \mathrm{HL}$ audiometric screening at octave frequencies $250-8000 \mathrm{~Hz}$. Participants were also screened for normal or corrected-tonormal visual acuity using a Snellen letter chart. Participants were asked to report any diagnoses of attention problems. Participants were compensated $\$ 10$ per hour. All testing was approved by the University of Louisville Internal Review Board.

Apparatus/materials. Participants were tested one at a time in a double-walled sound isolation chamber (Acoustic Systems). Each individual was presented with sounds over calibrated Beyerdynamic DT-990-Pro headphones, at moderate level (approximately $65 \mathrm{~dB}$ SPL). Software for the visual portion of the test was created using MATLAB.

Visual digit stimuli. Parameters of the digits portion of the test are identical to those reported in Experiment 1. 
Visuo-spatial Stimuli. Parameters of the visuo-spatial portion of the test are identical to those described in Experiment $2 \mathrm{a}$.

Auditory Stimuli. Auditory distracters were the same as those used in

Experiment 1. Durations of stimuli and interstimulus intervals were the same as Experiment 1.

Preliminary results from Experiment 1 also showed that the difficulty of the visual task, the sequence length, did not relate to the amount of ISE. For this reason, testing in Experiment $2 \mathrm{~b}$ took place over a restricted number of sequence lengths for each individual. These sequence lengths were determined by a span test, the details of which are described below.

Procedure. Digit Span test. Procedures for the digit span test were identical to those described in Experiment $2 a$.

Digits test with irrelevant sounds. Procedures of the digits test with irrelevant sound were the same as Experiment 1. However, participants were only tested for an ISE at their predetermined span. Each participant received one run of 20 trials in each of three distracter conditions; (a) quiet, (b) tones, and (c) letters.

Visuo-spatial span test. Procedures for the visuo-spatial span test were identical to those described in Experiment 2a.

Visuo-spatial test with irrelevant sounds. The form of a single trial was as follows, a cue appeared at the top of the screen indicating how many squares would be presented. Following this cue a number of squares were presented sequentially at random locations on the computer screen. Each square was present for $500 \mathrm{~ms}$, followed by a $500 \mathrm{~ms}$ pause. After the last square is presented the screen remained blank for a 
rehearsal period of $5 \mathrm{~s}$. Following the rehearsal period, all of the previously presented squares re-appeared. The individual was required to click on the squares in the order that they were presented. As each item is chosen they changed color. The participant had the option to change their answer. Once they clicked on every square, a "DONE" button appeared. They then received feedback, correct choices would turn to stars and incorrect choice would turn red. This task used a different set of spatial locations for every trial. Unlike other spatial tasks with fixed locations that can be given arbitrary names, this method ensured that there will be no verbal rehearsal. (See Figure 3.1 for a visual example of one trial)

Each run consisted of 15 trials, with 5 trials at each of three sequence lengths. Sequence lengths were determined by the visuo-spatial span test described above. Sequence lengths were randomly presented throughout the run. Each participant was tested at sequence lengths of Span, Span - 1 and Span +1 .

Distracter conditions were blocked. Participants completed 4 runs with each of the three distracter conditions for a total of 20 trials in each auditory condition. Blocks were randomly presented.

\section{Results}

Span data. Figure 3.3 displays a histogram showing the distribution of spans for the digit span task. Figure 3.4 displays the distribution of spans for the visuo-spatial span task. As found in Experiment 1, the average spans of this sample were also skewed towards higher spans relative to the norms (Orsini, et al., 1987). Since spans for Experiment 2 were determined using methods identical to those used by Orsini et al.,(1987), it is likely that these differences are instead due to sampling, and possibly to 
the education level of the participants. Relatively normal distributions can be observed on each of the two tasks. When plotted separately, the two age groups (adult and child) show similar distributions for both digit and visuo-spatial span, but with different means. Means and standard deviations for ages and spans are listed in Table 3.1. A significant positive correlation was found between age and span, $r(48)=.61, p<.01$.

Additionally, span data were analyzed to ensure that digit span and spatial span were related. In order to compare performance from one task to another, it is important that spans for these tasks show a strong relationship with one another. Figure 3.5 shows a scatter plot relating digit span to visuo-spatial span for all participants. There is a significant positive correlation between digit span and spatial span, $r(48)=.59, p<.001$. This ensures the appropriateness of further analyses which relate data collected at span for each task.

Comparison of ISE on digit task and visuo-spatial task. According to the phonological loop model, participants should show an ISE when tested on a verbal serial memory task, because of the disruption in rehearsal processes. However, participants should not show an ISE on a task which requires no verbal rehearsal. The AS data demonstrated that the visuo-spatial task does not require verbal rehearsal. Thus, it was predicted that individuals would not show an ISE on this task.

The dependent variable used to test the prediction made by the phonological loop model was the percent of correct trials on the visuo-spatial STM task and the digit test. These data were analyzed using only the data collected at the individual's predetermined span. Percent correct was entered into separate repeated measures 


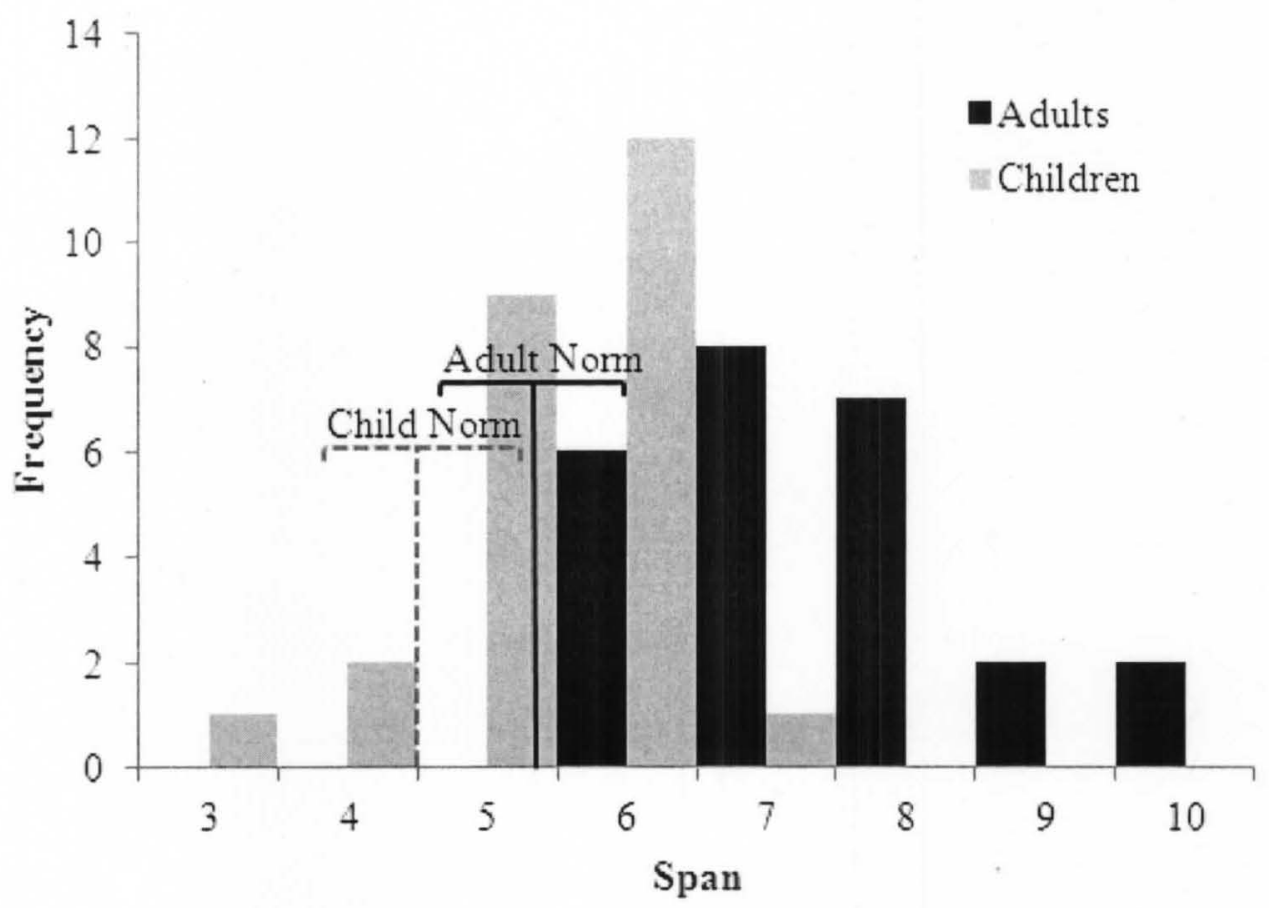

Figure 3.3. Span data for a digit span task is shown for adults and children. Digit span norms (Orsini et al., 1987) are indicated by the vertical lines, one standard deviation is indicated by the brackets (Adults, $M=5.4, S D=1.05$; Children, $M=4.5, S D=.95$ ). Spans were determined using a method that is similar to a standardized digit span test. Individuals were given two trials at each sequence length. Span was defined as the longest sequence length at which the individual could successfully replicate the digit sequence at least one out of two trials. Means and standard deviations for each group are found in Table 3.1. 


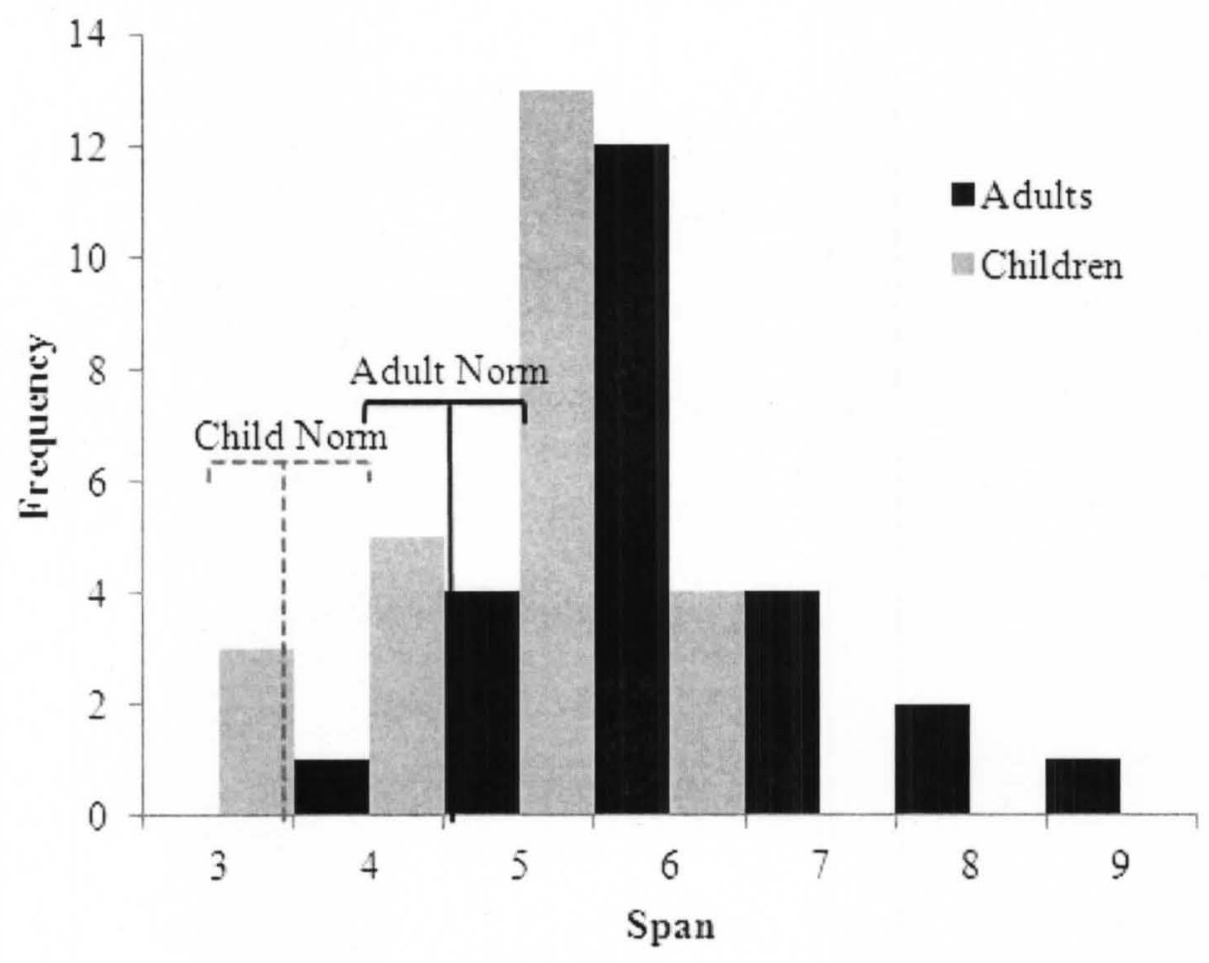

Figure 3.4. Span data for a visuo-spatial span task is shown for adults and children. Visuo-spatial span norms (Orsini et al., 1987) are indicated by the vertical lines, one standard deviation is indicated by the brackets (Adults, $M=4.6, S D=.75$; Children, $M=$ $3.9, S D=.9$ ). Spans were determined using a method that is similar to a standardized visuo-spatial span test. Individuals were given two trials at each sequence length. Span was defined as the longest sequence length at which the individual could successfully replicate the visuo-spatial sequence at least one out of two trials. Means and standard deviations for each group are found in Table 3.1. 
Table 3.1.

Summary of Ages and Spans of Groups

\begin{tabular}{cccc}
\hline Group & Mean Age $(S D)$ & \multicolumn{2}{c}{ Mean Span $(S D)$} \\
\hline & & Digit & Visuo-spatial \\
Adult & $24.3(6.0)$ & $7.4(1.1)$ & $6.2(1.1)$ \\
Child & $9.6(2.0)$ & $5.4(.9)$ & $4.7(.9)$ \\
\hline
\end{tabular}




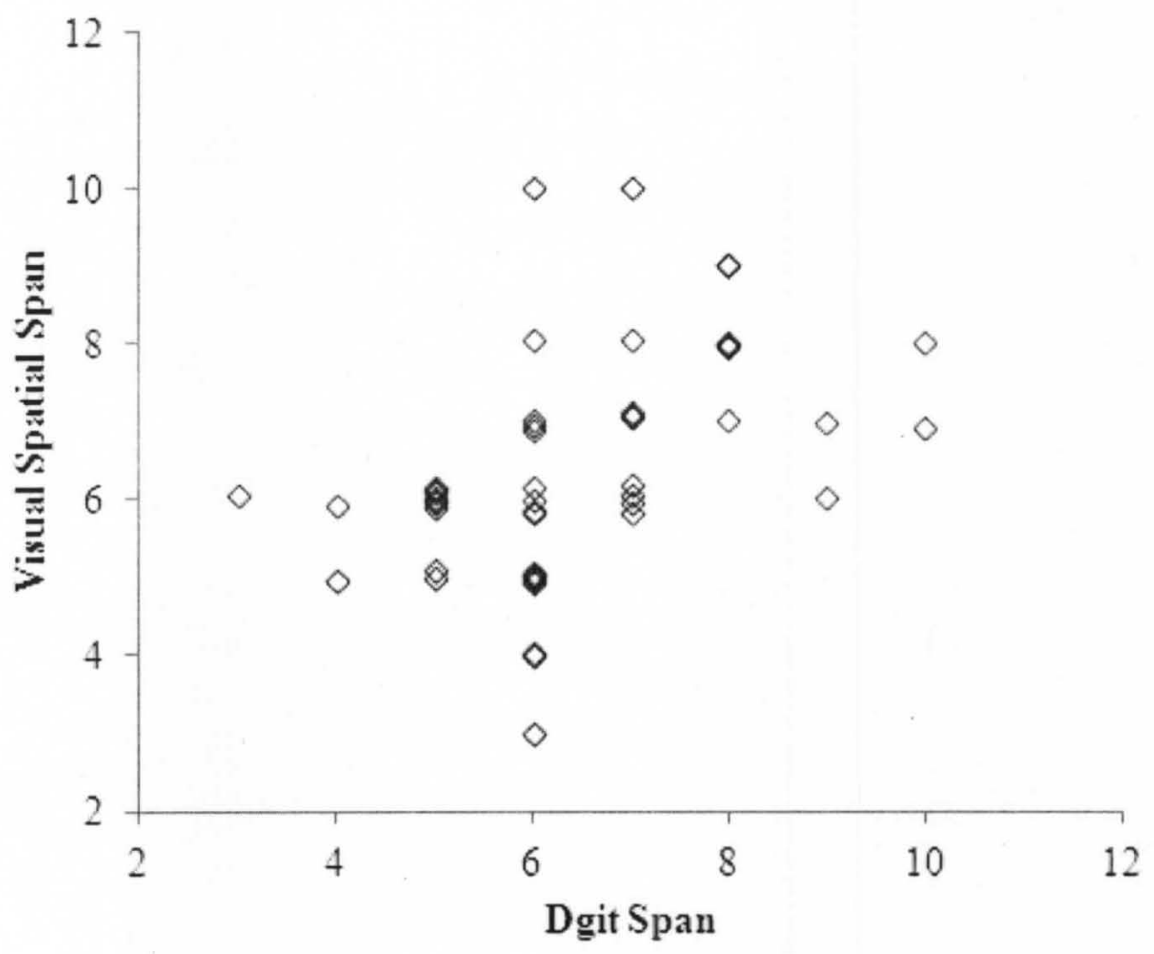

Figure 3.5. Relationship between visuo-spatial span and digit span. Scatter plot showing span data from all participants $(n=50)$. There is a significant positive correlation between digit span and visuo-spatial span $r(48)=.59, p<.001$. Visuo-spatial span data points have been jittered to prevent overlap. 
ANOVAs for each of the two memory tasks: digit and visuo-spatial. The within subjects factor was auditory condition with three levels: (a) quiet, (b) letters and (c) tones. An alpha level of 0.05 was used.

Data for the two memory tasks are shown in Figure 3.6. Results of this analysis for the digits task showed a significant main effect of condition, $F(2,98)=43.3, p<$ .001. Results of this analysis for the visuo-spatial task however, showed no significant main effects of condition $F(2,98)=.23, p=.794$. These results support predictions made by the phonological loop model. If a memory task requires no verbal rehearsal, the participant will not experience an ISE.

Relationship of age and memory task. There is a significant difference in memory capacity across age. As an individual gets older, their memory capacity increases. Similar to the within-age differences in capacity, these between age capacity differences may also be attributable to either attentional differences or rehearsal strategies. The second analysis in this study seeks to quantify the differences in ISE experienced between children and adults. Two questions are posed. First, will children show an increase in ISE relative to adults on a task which requires verbal rehearsal? If so, the results would support the hypothesis that differences in STM capacity are because of verbal rehearsal differences. And second, will children show the same increase in ISE on a task which requires no verbal rehearsal? If there is no increase, this is further evidence that the 


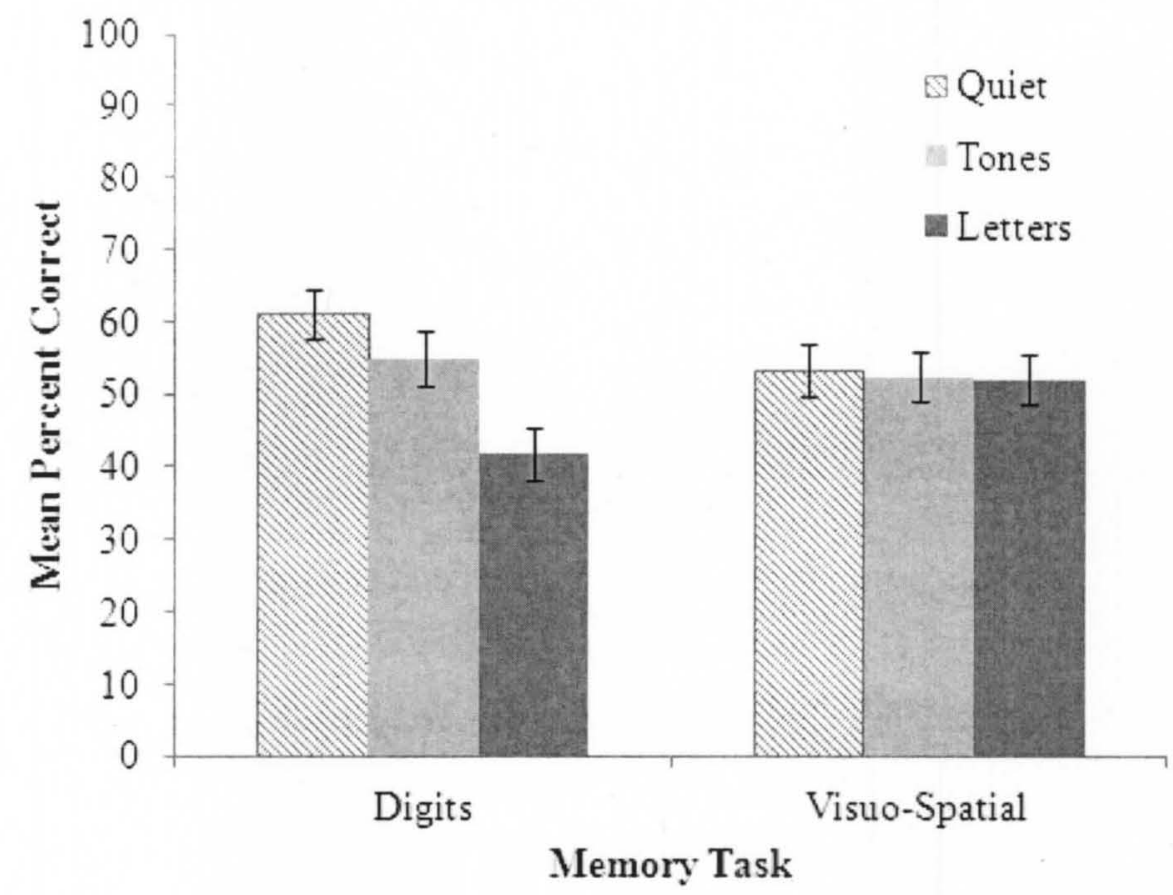

Figure 3.6. Mean performances for all participants $(n=50)$ on digit test and visuospatial test with the three auditory distracters. There was a significant effect of auditory distraction on the digits task, but no similar effect was demonstrated for the visuo- spatial task. Errors bars represent one standard error of the mean 
capacity differences are caused by rehearsal strategies and not to attentional differences.

The dependent variable analyzed was the percent of correct trials on the visuospatial STM task and the digit test. Percent correct was entered into separate mixed $2 \mathrm{x}$ 3 ANOVAs for each of the two memory tasks: digit and visual-spatial. The between subjects factor was age group, with two levels: child group and adult group. The within subjects factor was auditory condition with three levels: (a) quiet, (b) letters and (c) tones. An alpha level of 0.05 was used.

Figure 3.7 shows the result of the digits task by age group. Results of this analysis for the digits task indicated significant main effects of both condition, $F(2,98)$ $=45.13, p<.001$ and age, $F(2,98)=3.08, p=.003$. In addition, a significant interaction was found between the two factors $F(1,48)=3.086, p=.050$. Additional post hoc testing using Bonferroni correction (.05/4) revealed that there was a significant effect of condition between the quiet and letters conditions for the adults, $t(24)=-4.91, \mathrm{p}<.001$, and children, $t(24)=-7.67, p<.001)$ but no significant effect of condition was found between the quiet and tones condition for either age group: adults, $t(24)=-2.01, \mathrm{p}=$ .046 , children, $t(24)=-2.29, \mathrm{p}=.031$.

The significant interaction between age group and condition suggests that children are more adversely affected by irrelevant speech than adults. Neither group was significantly distracted by the non-speech materials, suggesting that these differential effects of distracter results from the disruption of the phonological loop.

Figure 3.8 shows the results of the visuo-spatial task by age group. Results of this analysis for the visuo-spatial data also support the predictions made by the 


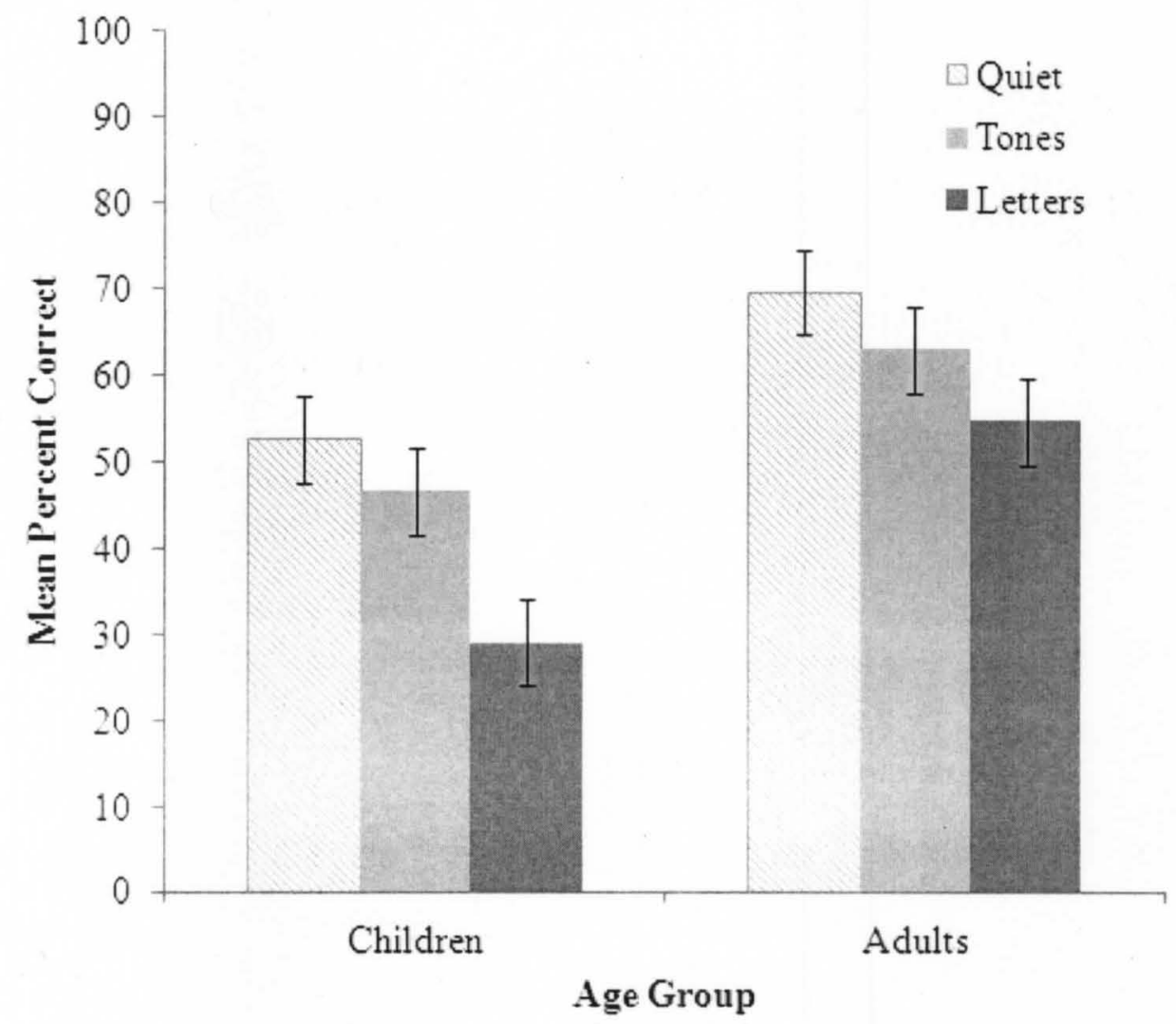

Figure 3.7. Data shown are the group mean performances for a serial memory digit task tested at span, with three auditory conditions. Data are separated by age group (Child group $n=25$, Adult group $n=25$ ). Each group shows a significant effect of the letters condition. Error bars represent one standard deviation from the mean. 


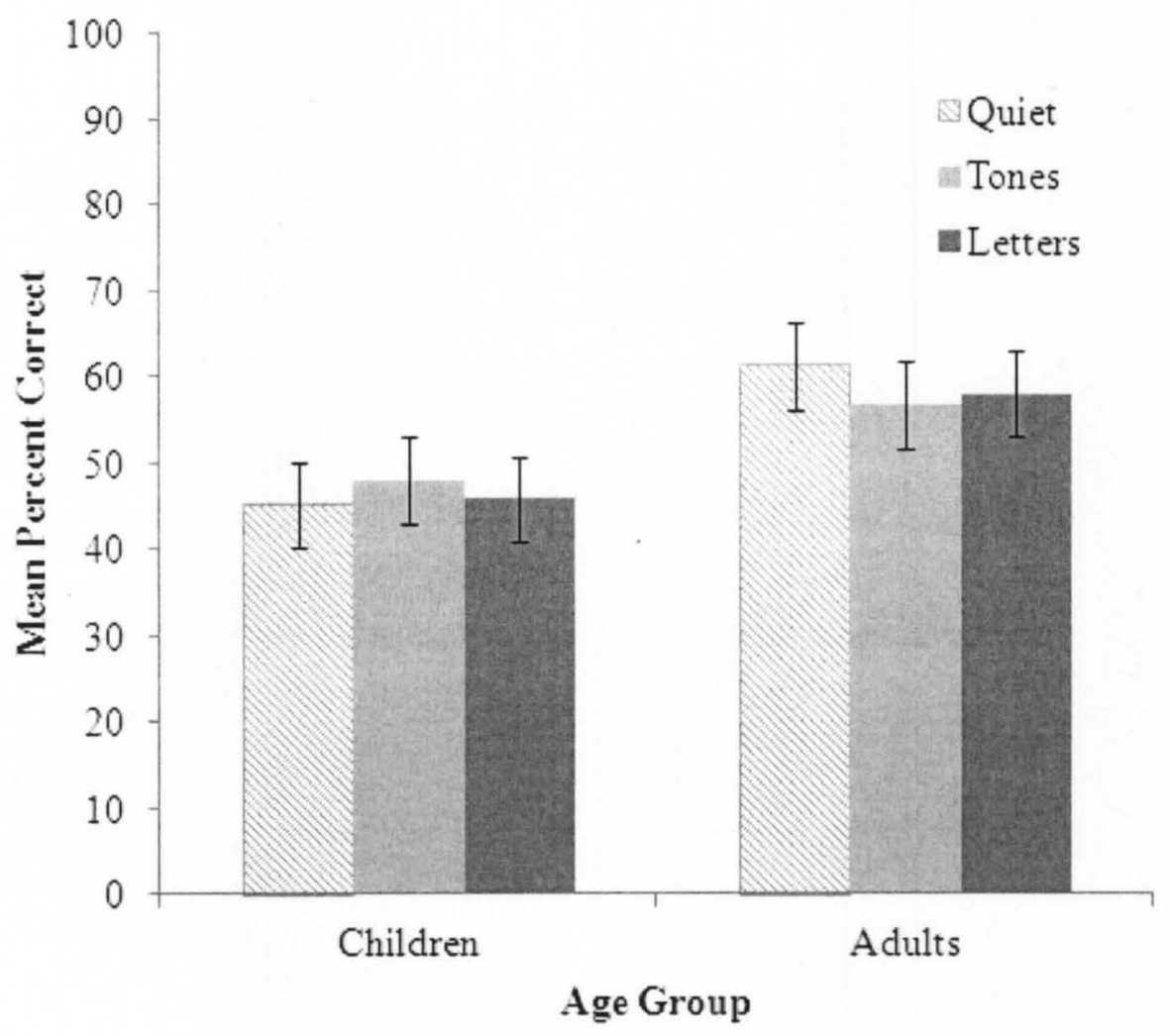

Figure 3.8. Data shown are the group mean performance for a serial memory visuospatial task tested at span with three auditory conditions. Data are separated by age group (Child group $n=25$, Adult group $n=25$ ). Neither group shows a significant effect of the letters or tones conditions. Error bars represent one standard error from the mean. 
phonological loop model. These data showed no significant main effects of condition $F(2,98)=.24, p=.791$, or age $F(2,98)=3.67, p=.061$, and no significant interaction between the two $F(1,48)=1.83, p=169$. Thus, when tested on a task which requires no verbal rehearsal, children perform similarly to adults. Their STMs are not adversely affected by irrelevant noise, because their capacity for the visuo-spatial materials is not determined by their rehearsal strategies. The non-speech condition (tones) is not significantly different from quiet. The child group also showed a greater disruption from speech (letters) than the adults

Span group comparisons. The previous analysis showed that individuals do not show an ISE when tested on a task that requires no overt rehearsal. Experiment 1 demonstrated that an individual's STM capacity could predict the amount of ISE they experienced. Individuals with reduced STM capacities were more affected by irrelevant speech than those with larger capacities. An individual's memory capacity may be determined by their rehearsal strategies, or their amount of attentional resources. Using the results from Experiement 1 alone would not be sufficient to determine where the source of the difference lies.. Using a nonverbal memory task helps to test the source of these differences. If the difference in capacity is determined by the attentional resources of the individual, and distraction is occurring because of a recruitment of these resources away from the relevant material, individuals with lower capacities should still demonstrate an ISE even with nonverbal materials. However, if the source of the difference in capacity is differences in rehearsal strategy, no difference between span groups should be observed on a task that requires no verbal rehearsal. 
In order to test this hypothesis, participants' data were split into four groups. Child and adult groups were each split into low and high span groups. See Table 3.2 for descriptions of the groups. These groups were determined in a similar manner to Experiment 1, using norms of STM. Unlike Experiment 1, however, there were not as many individuals with exceptionally high spans to warrant three span groups for the adults. Data were entered into a $2 \times 2 \times 3$ (Age Group [child, adult] x Span Group [high span, normal span] x Auditory Condition [quiet, tones, letters]) repeated measures ANOVA. Separate ANOVAs were run for each of the two memory tasks: digits and visuo-spatial. This analysis also used the percentage of correct trials as the dependent variable. Figure 3.9 shows the results of the digit task for each of the four span groups. For this task, the analysis revealed a significant main effect of age group $F(1,46)=$ $11.63, p=.001$ and span group, $F(1,46)=17.87, p<.001$. This significant main effect of span group was because the high span groups had an overall lower performance. A possible explanation for this outcome is that the high span individuals were operating at more difficult span lengths, which may not affect their ability to ignore distraction, but may produce an overall effect of a greater number of errors in every condition.

The analysis also revealed a significant main effect of auditory condition $F(2,92)=43.5, p<.001$, Although no interaction between the variables of condition and span group were observed, $F(1,62)=.484, p=.490$, post hoc analyses using Bonferroni correction (.05/8) examining the effects of condition by span group revealed a similar pattern of results to Experiment 1. See Table 3.3 for a summary of these 
Table 3.2.

Memory spans and group sizes for the four span groups

\begin{tabular}{lcc}
\hline Span Group & \multicolumn{2}{c}{ Memory Task } \\
\hline & Digit span $(n)$ & Visuo-spatial span $(n)$ \\
\cline { 2 - 3 } Normal Span child & $3-5(12)$ & $3-4(8)$ \\
High Span child & $6-7(13)$ & $5-6(17)$ \\
Normal Span Adult & $6-7(15)$ & $4-6(18)$ \\
High Span Adult & $8-10(10)$ & $7-9(7)$ \\
\hline
\end{tabular}




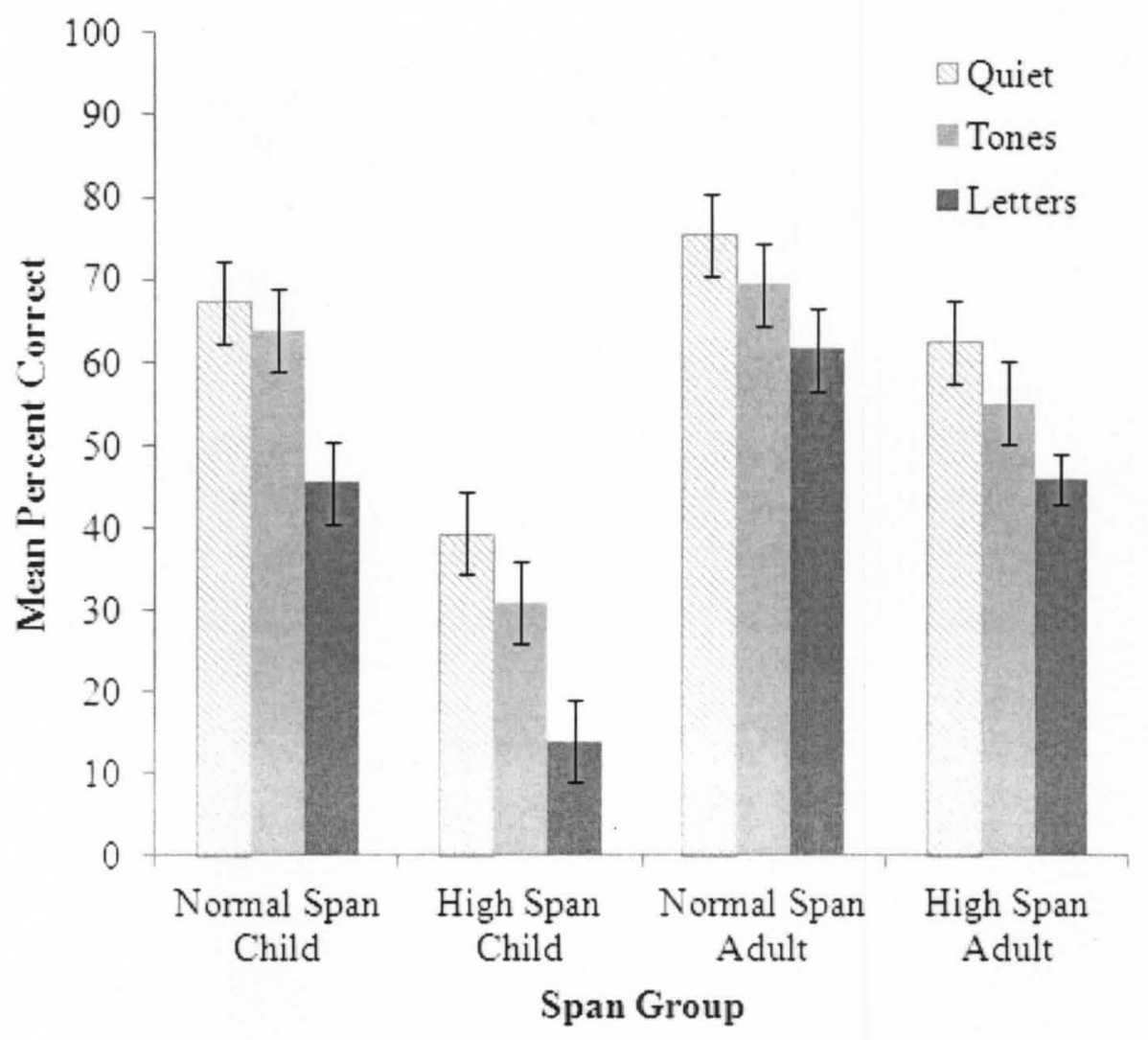

Figure 3.9. Span group means for the effects of auditory condition on a digit span task for lists at span only. All groups except high span adults show a significant effect of the letters condition. Error bars represent one standard error of the mean. 
Table 3.3 .

Summary of Post Hoc Analyses for Span Group Comparisons on Digit Task

\begin{tabular}{lccccc}
\hline Span Group & Comparison & $\boldsymbol{M}(\boldsymbol{S D})$ & $\boldsymbol{t}$ & $\boldsymbol{d} \boldsymbol{c}$ & $\boldsymbol{p}$ \\
\hline Normal span child & Q-T & $3.3(14.8)$ & 0.78 & 11 & .452 \\
& Q-L & $21.7(15.1)$ & 4.96 & 11 & $<.001$ \\
High span child & Q-T & $8.4(11.2)$ & 2.71 & 12 & .019 \\
& Q-L & $25.4(16.0)$ & 5.72 & 12 & $<.001$ \\
Normal span adult & Q-T & $6.1(11.8)$ & 1.92 & 13 & .076 \\
& Q-L & $13.9(13.4)$ & 3.87 & 13 & .002 \\
High span adult & Q-T & $7.3(20.1)$ & 1.19 & 10 & .259 \\
& Q-L & $16.4(17.9)$ & 3.03 & 10 & .013 \\
\hline
\end{tabular}

Note. $\mathrm{Q}-\mathrm{T}=$ Quiet to Tones comparison, $\mathrm{Q}-\mathrm{L}=$ Quiet to Letters comparison 
results. These analyses demonstrate two findings: (1) No group experienced an ISE from the non-speech auditory distracter. This is consistent with the results from Experiment 1. And (2) the high span adult group showed no significant ISE from either speech or non-speech distracter. This result is consistent with the findings from Experiment 1. A difference however was observed with the child data. The high span child group showed an effect of speech distracter. Possible explanations for this finding will be discussed further in the limitations section of the general discussion.

Figure 3.10 shows the results of the visuo-spatial task for each of the four span groups. For this task, there was no main effect of auditory condition, $F(2,92)=.044, p$ $=.957$. There was a significant main effect of span group, $F(1,46)=10.70, p=.002$. But the main effect of age group is no longer significant, $F(1,46)=1.00, p=.322$. Post hoc tests with Bonferroni correction revealed only a significant difference between the

high span child group and the normal span adult group $t(30)=-4.3 p=.005$. There was no interaction between span group and condition, $F(2,92)=.524, p=.594$. Given the lack of interaction on the digits task, these results are inconclusive. However, taken with the data from Experiment 1, which did show a relationship between span and ISE on a digits task, the results suggests that the difference in capacity is very likely to be attributable to rehearsal strategies and not attentional capacity.

\section{Discussion}

The pattern of results from Experiment 2 clearly shows that the ISE will not occur during a visuo-spatial serial memory task that requires no verbal rehearsal. Individuals 


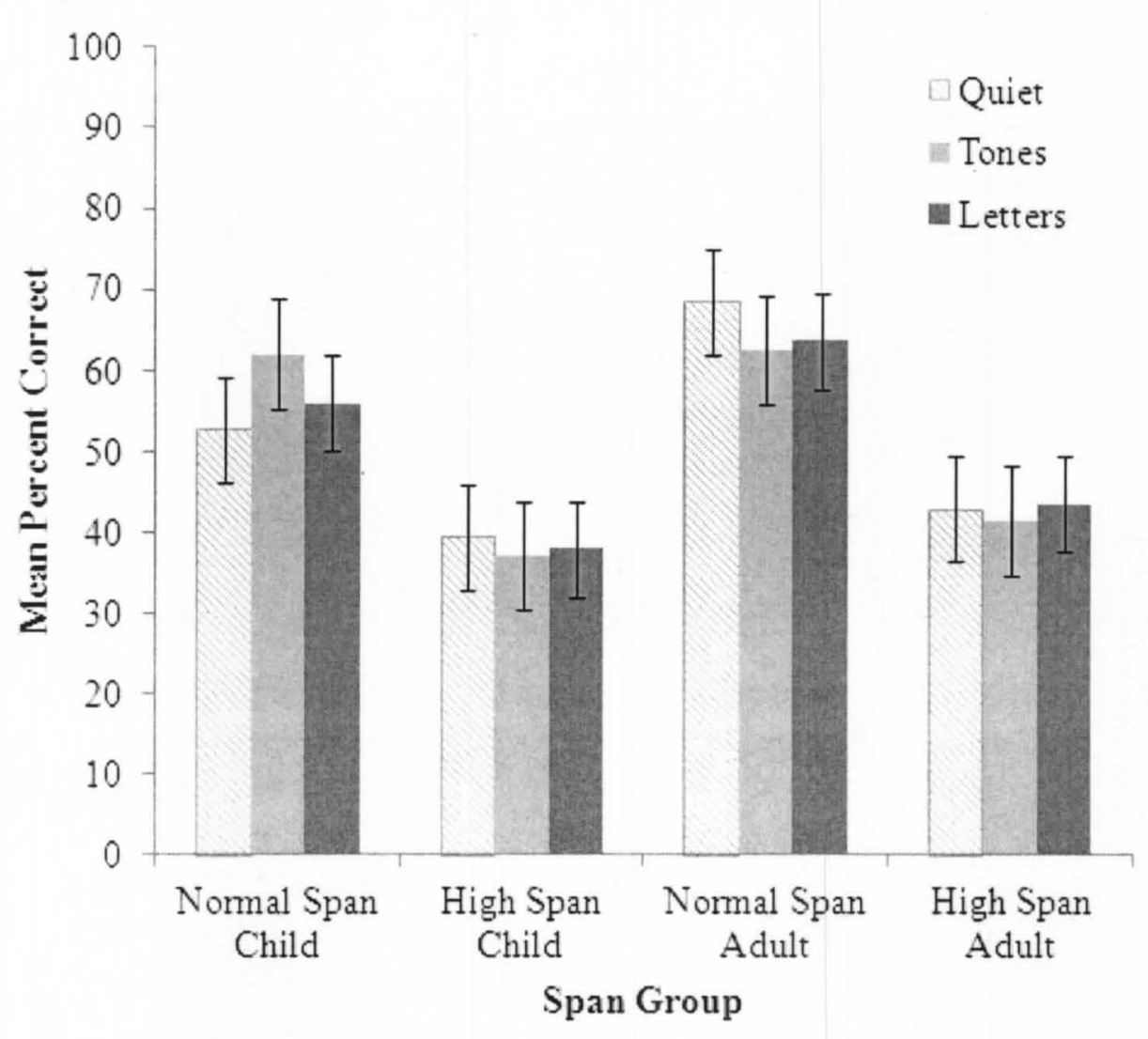

Figure 3.10. Span group means for the effects of auditory condition on a visuo-spatial span task for lists at span only. No group shows a significant effect of auditory condition. Error bars represent one standard error of the mean. 
showed no disruption of recall on the visuo-spatial task under conditions of auditory distraction. This finding supports predictions made by Baddeley's Model of Working Memory, which predicts that if a memory task requires no verbal rehearsal, there will be no disruption of recall by irrelevant speech. This prediction is inconsistent with Jones's changing state theory, which claims that there is a multi- modal ordering mechanism that organizes all incoming information, regardless of content. Jones's theory states that the ISE occurs because of the changing state nature of the distracter. However, the auditory distracters used in the current study were designed to have changing state components, and still they failed to elicit an ISE. Overall, these findings support the hypothesis that the ISE occurs because of a disruption in the phonological loop.

An additional finding of the current study was the lack of age effect on the visuospatial task. The results showed no main effect of age on the visuo-spatial task, demonstrating that the children performed as well as adults on the visuo-spatial task in the baseline condition (quiet). This was not the case for the digits task, which clearly showed a main effect of age. This lack of an age effect suggests that the process for retaining visuo-spatial material in STM is different than the process for retaining phonological material. This difference may lead not only to different developmental trajectories for digit memory and visuo-spatial memory, but also differences in susceptibility to ISE on these materials. Because the ISE reflects a disruption of verbal rehearsal strategies, one would expect children to show an ISE on a nonverbal serial memory task if capacity differences were determined by attentional factors rather than rehearsal factors. This was not the case in the current study. Children did not show poorer performance under conditions of auditory distraction with the visuo-spatial task. 
Thus, these results also support the hypothesis that developmental capacity differences are caused by rehearsal strategies, and not attentional strategies.

Finally, the span group analysis showed that individuals with lower spans did not show any evidence of an ISE with the visuo-spatial task. A similar conclusion can be made then with respect to individual differences in memory capacity as those made regarding developmental differences. Individuals with lower capacities appeared to show an increased susceptibility to distracters on a digit task in Experiment 1. However, no such result was found on a task with nonverbal materials. Consequently, it would appear that the individual differences in STM capacity may also be caused by rehearsal strategies rather than attentional differences. 


\section{CHAPTER IV}

\section{GENERAL DISCUSSION}

\section{Summary of the results}

The aim of the present study was to identify sources of individual and developmental differences on a task which is known to exhibit the ISE. These differences were investigated by manipulating the difficulty of the task (Experiment 1), by examining the nature of the task (Experiment 2), by examining the relationship between age and ISE (Experiments $1 \& 2$ ), and by examining the relationship between memory span and ISE (Experiments $1 \& 2$ ).

The results of Experiment 1 confirmed that an increase in the sequence length of the digit span task will not result in a greater ISE caused by a speech distracter. However, the results also indicated that the effects of a non-speech distracter are negated when controlling for the level of difficulty. Prior research has shown that individuals may switch encoding and rehearsal strategies with longer lists, perhaps relying more on a visual component of the to-be remembered materials rather than phonological coding (Baddeley \& Larsen 2007). This dependence on alternative processing strategies could potentially explain the results found by Jones and colleagues in which individuals experienced disruption by non-speech distracters (Jones \& Macken, 1993). 
Experiments 1 and 2 both demonstrated that if task difficulty is mediated, nonspeech auditory distracters do not cause an ISE. This finding supports the hypothesis that the ISE results from a disruption in the portion of memory that retains speech materials. Experiment 2 explored this hypothesis further by varying the nature of the STM task. Individuals were tested on a nonverbal serial memory task. These results clearly demonstrated that irrelevant auditory sounds had no effect on the nonverbal serial memory task. This was true for both speech and non-speech sounds. Taken together, these findings provide strong support for the hypothesis put forth by the Working Memory Model: The ISE results from disruption of phonological coding in the STM store.

In addition to investigating the effects of task difficulty and the nature of the task, developmental trends of the ISE were also examined. Results from Experiment 1 showed that when task difficulty is equated, children and adults are equally disrupted by speech distracters. However, this result was not replicated in Experiment 2. There was a slight, but significant interaction between age and auditory condition. This interaction occurred because the children experienced a greater interference by speech distracters. Past research has also reported mixed results with regards to this finding. Elliot (2002) found that children were more distracted by speech sounds than adults, while Klatte, et al. (2010) found that children and adults were equally distracted. However, in Elliot's study, only the youngest children displayed this effect. In the current study, the effect found in Experiment 2 was not restricted to the youngest children, but was dispersed among the age group. Therefore, the age restrictions for Experiment 2 are unlikely to be the primary source of the differences between the two experiments. 
There were two differences between Experiment 1 and Experiment 2 which may account for the differences in observed susceptibility. The first difference was methodological. In Experiment 1, individuals were tested at a range of sequence lengths. The data from the sequence length which resulted in performance closest to $50 \%$ correct was extracted and used for analysis. This procedure ensured that all individuals were evaluated at a similar level of difficulty. For Experiment 2, individuals were first given a span test, and then only tested at their span. This difference seems unlikely to have caused the difference in performance found between the two experiments, however, as the mean performance in quiet by the children in Experiment $2(52.6 \%)$ was similar to the performance in quiet in Experiment $1(59.4 \%)$. An independent samples $t$ test revealed no significant difference between these two group means, $t(44)=.239$. An additional difference between the two Experiments, which may have had a greater impact on the results, was the difference in mean spans. Figure 4.1 shows the distribution of spans for the children in Experiments 1 and 2. An independent samples t-test revealed a significant difference between these two groups $t(44)=2.13, p=.039$.. This difference in spans may have contributed to the differences in susceptibility found between the two experiments, particularly given the other data obtained from this study suggesting a link between ISE and memory span.

The results of Experiment 1 showed that an individual's memory span is a better predictor of individual differences in ISE than age. As demonstrated here, when an individual is performing at a high level of difficulty they will not necessarily experience greater amounts of ISE however, if an individual has a lower memory capacity, they may be more susceptible to distraction. This is a subtle distinction. Imagine two individuals 


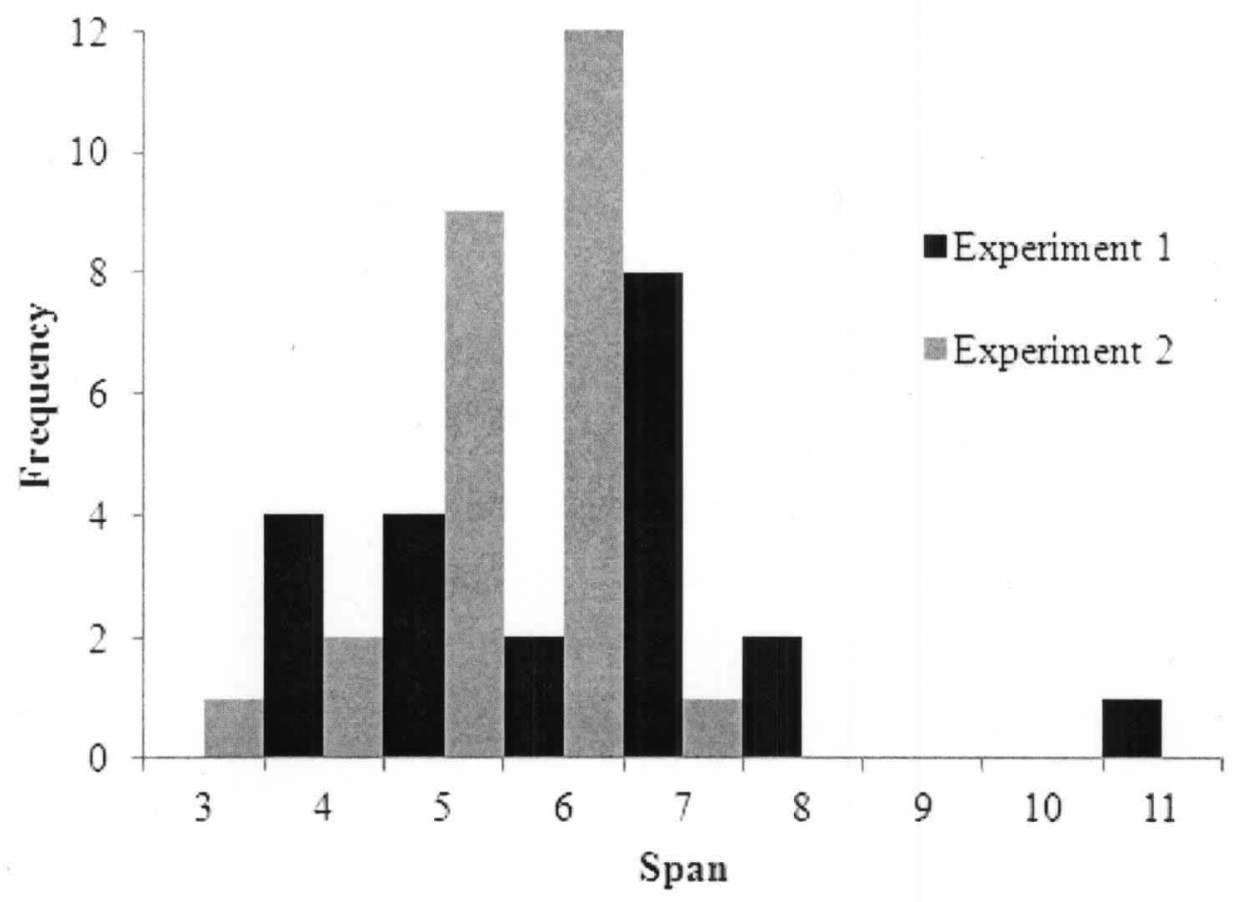

Figure 4.1. Data shown are spans of the children in each of the two experiments.

Experiment 1, $M=6.24, S D=1.73$, Experiment $2, M=5.40, S D=.87$. 
with different spans, Individual A has a short span, and therefore a more limited memory capacity. Individual B has a longer than typical span, and therefore a greater memory capacity. Individual A, when compared to Individual B will show a larger ISE when tested at baseline (span). However, neither individual will show an increase in the amount of ISE exhibited as the difficulty of the task increases. These effects are also independent of age. Children are not necessarily more susceptible to the ISE simply because they are younger, but they are more susceptible if they have lower memory capacities.

Similar span effects were not observed in Experiment 2, but this too may have been because of the large difference in mean span between the groups of participants in Experiment 1 versus Experiment 2. Experiment 1 had a greater number of high span individuals. Upon examination of the span distributions of the two experiments, it is clear that the sample in Experiment 1 is skewed towards containing a greater number of individuals with longer spans. This is true in both the child group and adult group. Experiment 2 contained a more typical distribution of spans, although still higher than normal. In the case of Experiment 1, significant differences were found between the high and normal span groups and the amounts of ISE experienced. When the data were analyzed by group, the high span individuals showed a reduced effect of auditory distraction. However, Experiment 2 had far fewer high span individuals. In order to examine span group differences most effectively, the population sample must contain individuals with very high spans and individuals with very low spans, and ideally a large number of each. This is difficult to obtain using a random sample of the population, as was demonstrated in these two experiments. Overall, the spans found in both 
experiments were higher than reported norms. As discussed in the previous sections, this was likely due to sampling differences, and educations levels of the samples. Future studies investigating these effects will need much larger sample sizes in order to detect these differences.

\section{Interpretations with regard to current theory}

The present results inform theory development regarding the ISE. There has been an ongoing debate between two competing theories that seek to explain this effect. The experiments in this thesis have directly tested predictions made by these two theories. The Working Memory Model predicts that (1) individuals will not experience an ISE when presented with nonverbal auditory distractions, and (2) individuals will not experience an ISE when tested on a nonverbal serial memory task (Salamè \& Baddeley, 1982). The changing state theory makes the opposite prediction. This theory posits that the content of the distracter in unimportant, only that it is changing state (Jones \& Macken, 1993). Furthermore, the changing state theory predicts that an individual will still experience an ISE even on a test of visuo-spatial STM (Tremblay, Parmentier, Guerard, Nicholls, \& Jones, 2006).

The results of the current experiments support the first prediction made by the working memory model. Results clearly indicate a relationship between the content of the distracter and the amount of distraction experienced. The non-speech auditory distracters in the current study were designed to conform to the changing state criteria by varying both in frequency and tempo. By all accounts, if the changing state theory is correct, these non-speech materials should have exhibited an ISE. However, no such 
results were found. The results also support the second prediction made by the working memory model. No effect of distraction was observed on the nonverbal serial memory task.

The working memory model posits that STM capacity is largely determined by both coding strategies (for example, verbal rehearsal) and active attention. In addition, coding strategies and attention are also linked (Engle et al., 1999). Coding strategies may be more or less attention demanding depending on the individual. If the coding strategy is more demanding on attention, attention is recruited away, and intrusions from irrelevant materials can occur. The age of the individual may affect how demanding the coding strategy will be on attention (Engle et al., 1999). Perhaps these strategies are newly learned, and not yet automatic. It follows then that those individuals with the most difficulty with coding strategies will show a greater ISE. It is possible that phonological coding is more attentionally demanding than the coding strategy used for visuo-spatial materials and for this reason the ISE does not occur on a task with visuo-spatial materials.

\section{Implications of research}

Because a great deal of learning takes place in childhood and adolescence, it is critical to understand how noise can potentially affect children's ability to learn. The effects of moderate intensity noise on learning in children have not yet been thoroughly investigated. These types of noise could come from inside the classroom in the form of classmates' conversations, announcements, etc. There may also be non-speech environmental noises such as furniture moving, computer noise, heating and cooling. 
One goal of this study was to investigate whether different types of noise have differential effects on specific types of cognitive processes involved in children's learning.

Field observations of classroom noise have shown that cognitive tasks can be affected. Thus, laboratory tests of the acute effects of specific types of noise on cognitive abilities of children should receive further investigation. The current study investigated the effects of noise on STM. STM is a necessary component of learning. The ability to hold verbal items in STM is a predictor of children's vocabulary (Baddeley, Gathercole, \& Papagno, 1998), is associated with expressive language development (Adams, 1996), storage of sentences in memory (Gathercole, Willis, Emslie, \& Baddeley, 1992), listening comprehension (Dufva, Niemi, \& Voeten, 2001), and reading achievement (Baddeley \& Gathercole, 1993). STM has been shown in adults to be highly sensitive to the negative effects of moderate intensity noise (Jones, Hughes, Marsh, \& Macken, 2008; Macken, Phelps, \& Jones, 2009). However, the effect of moderate intensity noise on children's STM is not well understood. Therefore, this study serves to fill a gap in the literature, and provides motivation for future investigations into the effects of noise on children's learning. The current study demonstrated that irrelevant speech sounds have a detrimental effect on children's performance on STM tasks that involve phonological processing. Therefore, teachers and parents should take measures to provide quiet working environments for children when they are engaged in these types of tasks, such as language learning, or reading comprehension.

A predictive factor of distractibility, such as memory capacity, would be useful in 
determining which children would be most affected by noise in the classroom. The current research has demonstrated that memory capacity may be an important factor in distractibility. It is possible that an individual's encoding efficiency affords them greater cognitive resources to devote to inhibition of distraction.

\section{Future Research}

The ISE has been shown mainly to affect recall for serial STM tasks, such as digit span. Therefore, in order to make a stronger argument for the effects of irrelevant speech on learning in the classroom, direct tests should be made between learning tasks, such as reading comprehension, or word learning, and the effects of irrelevant speech. Klatte et al. (2007) examined the effect of irrelevant speech on the memorization of non-words in children and found that performance was significantly degraded under these conditions. Similar studies using learning tasks such as those seen in a classroom would help to further clarify the effect.

Several interesting questions arise with respect to the findings of Experiment 2. An individual's ability to inhibit incoming information may be related to their STM capacity. However, visuo-spatial STM capacity does not seem to have the same relationship when tested with auditory distracters. If the coding strategies for visuospatial materials in STM require less attention, are there still ways to disrupt these strategies? Past research suggests that manual tapping may disrupt recall for visuo-spatial materials (Farmer, Berman \& Fletcher, 1986). Would visuo-spatial span differences then be predictive of the amount of interference by this type of task? As yet, no studies have 
examined the relationship between visuo-spatial STM capacity and ability to inhibit nonauditory distraction.

\section{Conclusions}

Overall, the results of this series of experiments are consistent with Baddeley's Model of Working Memory, and do not support the changing state theory proposed by Jones. Results indicate that the ISE is caused only by speech distracters, and then only during a STM task which requires active verbal rehearsal. These effects of irrelevant speech were found in both children and adults, provided that sequence lengths are adjusted to equate for performance. Additionally, there appears to be a negative relationship between STM capacity and the magnitude of the ISE in both children and adults, the smaller the STM capacity, the larger the ISE. 


\section{REFERENCES}

Adams, A. M. (1996). Phonological working memory and spoken language development in young children. The Quarterly Journal of Experimental Psychology: Section A, 49(1), 216-233.

Baddeley, A. D. (1972). Retrieval rules and semantic coding in short-term memory. Psychological Bulletin, 78(5), 379-385. doi:10.1037/h0033477

Baddeley, A. D. (1983). Working memory. Philosophical transactions of the royal society of london. Series B, Biological Sciences 1, 302(1110), 311-324.

Baddeley, A. D., \& Gathercole, S. E. (1993). Working Memory and Language. Hillsdale, NJ: Lawrence Erlbaum Associates Publishers.

Baddeley, A. D., \& Hitch, G. J. (2000). Development of working memory: should the Pascual-Leone and the Baddeley and Hitch models be merged? Journal of experimental child psychology, 77(2), 128-37. doi:10.1006/jecp.2000.2592

Baddeley, A. D., \& Larsen, J. D. (2003). The disruption of STM: A response to our commentators. The Quarterly Journal of Experimental Psychology: Section A, 56(8), 1301-1306. doi:10.1080/02724980343000530

Baddeley, A. D., \& Larsen, J. D. (2007). The phonological loop unmasked? A comment on the evidence for a "perceptual-gestural" alternative. Quarterly journal of experimental psychology (2006), 60(4), 497-504.

doi: $10.1080 / 17470210601147572$ 
Baddeley, A. D., Gathercole, S. E., \& Papagno, C. (1998). The phonological loop as a language learning device. Psychological review, 105(1), 158-73.

Baddeley, A. D., Lewis, V., \& Vallar, G. (1984). Exploring the articulatory loop. The Quarterly Journal of Experimental Psychology Section A, 36(2), 233-252.

Baddeley, A. D., Thomson, N., \& Buchannon, M. (1975). Word length and the structure of short-term memory. Journal of Verbal Learning and Verbal Behavior, 14(6), 575-589. doi:10.1016/S0022-5371(75)80045-4

Beaman, C. P. (2004). The irrelevant sound phenomenon revisited: what role for working memory capacity? Journal of Experimental Psychology: Learning, Memory, and Cognition, 30(5), 1106-1118.

Bridges, A., \& Jones, D. (1996). Word dose in the disruption of serial recall by irrelevant speech: phonological confusions or changing state? The Quarterly Journal of Experimental Psychology A, 49(4), 919-939.

doi: $10.1080 / 027249896392360$

Case, R., \& Globerson, T. (1974). Field independence and central computing space.Child Development, 45(3), 772. doi:10.2307/1127844

Case, R., Kurland, M., \& Goldberg, J. (1982). Operational efficiency and the growth of short-term memory span. Journal of Experimental Child Psychology, 33(3), 386404. doi:10.1016/0022-0965(82)90054-6

Colle, H. A., \& Welsh, A. (1976). Acoustic masking in primary memory. Journal of Verbal Learning \& Verbal Behavior, 15(1), 17-31. doi:http://dx.doi.org/10.1016/S0022-5371\%2876\%2990003-7

Conway, A. R., Cowan, N., \& Bunting, M. F. (2001). The cocktail party phenomenon 
revisited: the importance of working memory capacity. Psychonomic bulletin \& review, 8(2), 331-5.

Corsi, P. (1972). Human memory and the medial temporal region of the brain. Dissertation Abstracts International: 34(2-B), 891.

Cowan, N. (1995). Attention and memory: An integrated framework. Oxford psychology series, No. 26. New York, Oxford University Press.

Dempster, F. N. (1981). Memory span: Sources of individual and developmental differences. Psychological Bulletin, 89(1), 63-100.

Doyle, A. B. (1973). Listening to distraction: a developmental study of selective attention. Journal of experimental child psychology, 15(1), 100-15.

Dufva, M. I. A., Niemi, P., \& Voeten, M. J. M. (2001). The role of phonological memory, word recognition , and comprehension skills in reading development: from preschool to grade 2. Reading, (1990), 91-117.

Ellermeier, W., \& Hellbrück, J. (1998). Is level irrelevant in “irrelevant speech”? Effects of loudness, signal-to-noise ratio, and binaural unmasking. Journal of Experimental Psychology: Human Perception and Performance, 24(5), 1406-1414. doi:http://dx.doi.org/10.1037/0096-1523.24.5.1406

Ellermeier, W., \& Zimmer, K. (1997). Individual differences in susceptibility to the "irrelevant speech effect." Journal of the Acoustical Society of America, 102(4), 2191-2199. doi:http://dx.doi.org/10.1121/1.419596

Elliott, E. M. (2002). The irrelevant-speech effect and children: theoretical implications of developmental change. Memory and Cognition, 30(3), 478-487.

Elliott, E. M., \& Cowan, N. (2005). Coherence of the irrelevant-sound effect: 
individual profiles of short-term memory and susceptibility to task-irrelevant materials. Memory and Cognition, 33(4), 664-675.

Elliott, E. M., Barrilleaux, K., \& Cowan, N. (2006). Individual differences in the ability to avoid distracting sounds. European Journal of Cognitive Psychology, 18(1), 90-108. doi:10.1080/09541440500216044

Engle, R. (2002). Working memory capacity as executive attention. Current Directions in Psychological Science, 11(1), 19-23. doi:10.1111/1467-8721.00160

Engle, R., Tuholski, S. W., Laughlin, J. E., \& Conway, A. R. (1999). Working memory, short-term memory, and general fluid intelligence: A latent-variable approach. Journal of Experimental Psychology: General, 128(3), 309.

Evans, G. W., \& Lepore, S. J. (1993). Nonauditory effects of noise on children: a critical review. Childrens Environments, 10(1), 31 .

Farmer, E. W., Berman, J. V. F., \& Fletcher, Y. L. (1986). Evidence for a visuo-spatial scratch-pad in working memory. The Quarterly Journal of Experimental Psychology Section A, 38(4), 675-688.

Gathercole, S. E., Willis, C. S., Emslie, H., \& Baddeley, A. D. (1992). Phonological memory and vocabulary development during the early school years: A longitudinal study. Developmental Psychology, 28(5), 887-898.

Gomes, H., Molholm, S., Christodoulou, C., Ritter, W., \& Cowan, N. (2000). The development of auditory attention in children. Frontiers in bioscience: a journal and virtual library, 5, D108-20.

Hale, S., Bronik, M. D., \& Fry, a F. (1997). Verbal and spatial working memory in school-age children: developmental differences in susceptibility to interference. 
Developmental psychology, 33(2), 364-71. Retrieved from

http://www.ncbi.nlm.nih.gov/pubmed/9147843

Hetu, R., Truchon-Gaganon, C., \& Bilodeau, S. A. (1990). Problems of noise in school settings: A review of literature and the results of an exploratory study. Journal of Speech Language Pathology and Audiology, 14(3), 31-39.

Hofferth, S. L., Smith, J., McLoyd, V. C., \& Finkelstein, J. (2000). Achievement and Behavior Among Children of Welfare Recipients, Welfare Leavers, and LowIncome Single Mothers. Journal of Social Issues, 56(4), 747-774.

doi:10.1111/0022-4537.00194

Jones, D. (1993). Objects, streams and threads of auditory attention. In A. D. Baddeley \& L. Weiskrantz $\left(1^{\text {st }}\right.$ Ed), Attention: Selection, Awareness, and Control A tribute to Donald Broadbent (pp. 87-104). New York: Oxford University Press.

Jones, D., \& Macken, W. (1993). Irrelevant tones produce an irrelevant speech effect: Implications for phonological coding in working memory. Journal of Experimental Psychology: Learning, Memory, and Cognition, 19(2), 369-381. doi:http://dx.doi.org/10.1037/0278-7393.19.2.369

Jones, D., \& Macken, W. (1995a). Phonological similarity in the irrelevant speech effect: within-or between-stream similarity? Journal of Experimental Psychology: Learning, Memory and Cognition, 21, 103-103.

Jones, D., \& Macken, W. (1995b). Organizational factors in the effect of irrelevant speech: the role of spatial location and timing. Memory and Cognition, 23(2), 192-200.

Jones, D., Alford, D., Bridges, A., Tremblay, S., \& Macken, W. (1999). Organizational 
factors in selective attention: The interplay of acoustic distinctiveness and auditory streaming in the irrelevant sound effect. Journal of Experimental Psychology: Learning, Memory, and Cognition, 25(2), 464.

Jones, D., Farrand, P., Stuart, G., \& Morris, N. (1995). Functional equivalence of verbal and spatial information in serial short-term memory. Learning, Memory, 21(4), 1008-1018.

Jones, D., Hughes, R., \& Macken, W. (2007). The phonological store abandoned. TheQuarterly Journal of Experimental Psychology A: Human Experimental Psychology,60(4), 505-511.

Jones, D., Hughes, R., \& Macken, W. (2010). Auditory distraction and serial memory: The avoidable and the ineluctable. Noise and Health, 12(49), 201.

Jones, D., Hughes, R., Marsh, J. E., \& Macken, W. (2008). Varieties of auditory distraction. Proceedings of ICBEN, 362-368.

Jones, D., Macken, W., \& Mosdell, N. A. (1997). The role of habituation in the disruption of recall performance by irrelevant sound. British Journal of Psychology,88(4), 549-564.

Jones, D., Macken, W., \& Murray, A. C. (1993). Disruption of visual short-term memory by changing-state auditory stimuli: the role of segmentation. Memory and Cognition, 2l(3), 318-328.

Jones, D., Macken, W., \& Nicholls, A. P. (2004). The phonological store of working memory: is it phonological and is it a store? Journal of Experimental Psychology: Learning, Memory, and Cognition, 30(3), 656-674. doi:http://dx.doi.org/10.1037/0278-7393.30.3.656 
Jones, D., Madden, C., \& Miles, C. (1992). Privileged access by irrelevant speech to short-term memory: The role of changing state. The Quarterly Journal of Experimental Psychology A: Human Experimental Psychology, 44A(4), 645669.

Jones, D., Miles, C., \& Page, J. (1990). Disruption of proofreading by irrelevant speech: Effects of attention, arousal or memory? Applied Cognitive Psychology, $4(2), 89-108$.

Kane, M. J., \& Engle, R. (2003). Working-memory capacity and the control of attention: The contributions of goal neglect, response competition, and task set to Stroop interference. Journal of Experimental Psychology: General, 132(1), 47-70. doi:10.1037/0096-3445.132.1.47

Kane, M. J., Bleckley, M. K., Conway, A. R., \& Engle, R. (2001). A controlledattention view of working-memory capacity. Journal of experimental psychology. General, 130(2), 169-83.

Klatte, M., Lachmann, T., Schlittmeier, S. J., \& Hellbrück, J. (2010). The irrelevant sound effect in short-term memory: Is there developmental change? European Journal of Cognitive Psychology, 22(8), 1168-1191. doi:10.1080/09541440903378250

Klatte, M., Meis, M., Sukowski, H., \& Schick, A. (2007). Effects of irrelevant speech and traffic noise on speech perception and cognitive performance in elementary school children. Noise and Health, 9(36), 64.

Lane, D. M., \& Pearson, D: A. (1982). The development of selective attention. MerrillPalmer Quarterly: Journal of Developmental Psychology. 28(3), 317-337. 
Larsen, J. D., \& Baddeley, A. (2003). Disruption of verbal STM by irrelevant speech, articulatory suppression, and manual tapping: do they have a common source? The Quarterly journal of experimental psychology. A, Human experimental psychology, 56(8), 1249-68. doi:10.1080/02724980244000765

LeCompte, D., \& Shaibe, D. (1997). On the irrelevance of phonological similarity to the irrelevant speech effect. The Quarterly Journal of Experimental psychology, 50A(1), 100-118. doi:10.1080/713755679

LeCompte, D., Neely, C. B., \& Wilson, J. R. (1997). Irrelevant speech and irrelevant tones: the relative importance of speech to the irrelevant speech effect. Journal of experimental psychology. Learning, memory, and cognition, 23(2), 472-83.

Logie, R., \& Pearson, D. (1997). The inner eye and the inner scribe of visuo-spatial working memory: evidence from developmental fractionation. European Journal of Cognitive Psychology, 9(3), 241-257. doi: 10.1080/713752559.

Macken, W., Mosdell, N. A., \& Jones, D. (1999). Explaining the irrelevant-sound effect: Temporal distinctiveness or changing state? Journal of Experimental Psychology: Learning, Memory, and Cognition, 25(3), 810-814. doi:http://dx.doi.org/10.1037/0278-7393.25.3.810

Macken, W., Phelps, F. G., \& Jones, D. (2009). What causes auditory distraction? Psychonomic bulletin \& review, 16(1), 139-44. doi:10.3758/PBR.16.1.139 Macken, W., Tremblay, S., Houghton, R. J., Nicholls, A. P., \& Jones, D. (2003). Does auditory streaming require attention? Evidence from attentional selectivity in shortterm memory. Journal of Experimental Psychology: Human Perception and Performance, 29(1), 43-51. doi:http://dx.doi.org/10.1037/0096-1523.29.1.43 
Miller, G. A. (1956). The magical number seven, plus or minus two: some limits on our capacity for processing information. Psychological Review, 63(2), 81-97. doi: $10.1037 / \mathrm{h} 0043158$

Orsini, A., Grossi, D., Capitani, E., Laiacona, M., Papagno, C., \& Vallar, G. (1987). Verbal and spatial immediate memory span: normative data from 1355 adults and 112 children. Italtian Journal of Neurological Sciences, 8(6), 539-548.

Salamé, P., \& Baddeley, A. D. (1982). Disruption of short-term memory by unattended speech: Implications for the structure of working memory. Journal of Verbal Learning \& Verbal Behavior, 2l(2), 150-164. doi:http://dx.doi.org/10.1016/S0022$5371 \% 2882 \% 2990521-7$

Salamé, P., \& Baddeley, A. D. (1986). Phonological factors in STM: Similarity and the unattended speech effect. Bulletin of the Psychonomic Society, 24(4), 263-265.

Salamé, P., \& Baddeley, A. D. (1989). Effects of background music on phonological short-term memory. The Quarterly Journal of Experimental Psychology A: Human Experimental Psychology, 41(1-A), 107-122.

Shield, B. M., \& Dockrell, J. E. (2003). The effects of noise on children at school: a review. Building Acoustics, 10(2), 97-116.

Turner, M. L., \& Engle, R. (1989). Is working memory capacity task dependent? Journal of Memory and Language, 28(2), 127-154. doi:http://dx.doi.org/10.1016/0749$596 X \% 2889 \% 2990040-5$

Tremblay, S., Nicholls, A. P., Alford, D., \& Jones, D. (2000). The irrelevant sound effect: does speech play a special role? Journal of Experimental Psychology: Learning, Memory, and Cognition, 26(6), 1750-1754. 
Tremblay, S., Parmentier, F. B., Guerard, K., Nicholls, A. P., \& Jones, D. (2006). A spatial modality effect in serial memory. Journal of Experimental Psychology: Learning, Memory, and Cognition, 32(5), 1208-1215. doi:http://dx.doi.org/10.1037/0278-7393.32.5.1208

Wood, C. (2002). Parent-child pre-school activities can affect the development of literacy skills. Journal of Research in Reading, 25(3), 241-258. doi:10.1111/14679817.00173 
CURRICULUM VITAE

\begin{abstract}
AMANDA O'BRYAN
Department of Psychological and Brain Sciences

University of Louisville

4536 Southern Parkway

Heuser Hearing Research Center

Louisville, KY 40214

$502-442-4293$

111 E. Kentucky St.

afobry01@louisville.edu

Louisville, KY 40203

502-852- 3860
\end{abstract}

\title{
EDUCATION
}

Ph.D. Experimental Psychology

M.S. Experimental Psychology
University of Louisville

Focus: Auditory Perception

Expected Summer 2012

University of Louisville

Focus: Auditory Perception

2009

College of Charleston

1999

\section{B.S. Psychology}

\section{ACADEMIC AWARDS/SCHOLARSHIPS}

Kentuckiana Metroversity Outstanding Faculty (nominee) 2012

University of Louisville Tuition Award

2010-2011

College of Charleston, LIFE Scholarship

1997-199 


\section{TEACHING EXPERIENCE}

Instructor, Jefferson Community and Technical College

2010-2011

- Introductory Psychology

- Developmental Psychology

Teaching Assistant, University of Louisville

2007-2009

- Served as T.A. for undergraduate courses: Introduction to Psychology (2007) and Experimental Psychology (2008)

- Enhanced student understanding of material by leading discussion sections, holding office hours, answering emails and participating in review sessions and study groups

- Created lab experiments and graded exams and assignments

Workshop organizer, University of Louisville

$2010 \& 2011$

- Organized and managed the "Technology for Effective Teaching" workshop for the School of Interdisciplinary and Graduate Studies

Graduate Teaching Academy, University of Louisville

2008

\section{TEACHING INTERESTS}

- Sensation and Perception

- Statistics

- Developmental Psychology

- Research Methods

- Experimental Psychology

- Introduction to Psychology

\section{RESEARCH EXPERIENCE}

Doctoral Research: Department of Psychological and Brain Sciences, UofL 
2009-present

- Advisor: Dr. Pavel Zahorik

- The Irrelevant speech effect in children and adults and its relationship to cognitive factors such as working memory capacity and attention

- Selected technical skills include: programming experiments and creating stimuli using Matlab, calibrating equipment, and designing sound environments

Graduate Research Assistant, Department of Psychological and Brain Sciences, UofL 2009-2010

- Release from informational masking using dichotic presentation in children and adults

Lab Technician, Heuser Hearing Research Center Summer 2008, 2009

- Assisted in testing both normally hearing children and children with cochlear implants during the Heuser Hearing Research Center Summer Science Camp

- Investigated the development of auditory processing using psychophysical techniques

Lab Manager, Heuser Hearing Research Center

2007-2008

- Handled recruiting, scheduling, testing, and payment of human subjects

\section{UNIVERSITY AND ACADEMIC SERVICE}

Association for Psychological Science, 2010, 2011

- Reviewer for the 2010 APS Student Grant Competition

- Reviewer for the 2011 APS Student Research Award

Assistant to the Psychology Undergraduate Chair, 2009-2010

- Helped to compile a database of undergraduate psychology student information

Experimental Psychology Graduate Student Seminar, 2008-2009

- Organized and managed a departmental colloquium series for the Experimental Psychology Graduate students

Representative 2007-2008 
- Elected Psychology Graduate student representative

\section{CLINICAL EXPERIENCE}

Psychosocial Rehabilitation Counselor, Alliance Family Services, Sandpoint, Idaho, 2001-2003

- Worked in the homes and schools of at-risk youth using behavior modification therapies, filed progress reports

Residential Counselor, Maple Manor, Charleston, SC, 2000-2001

- Counselor to adult schizophrenics working towards assisted living, prepared medication, transported patients

\section{PUBLICATIONS}

O'Bryan, A., Individual and Developmental differences in the susceptibility to the Irrelevant Speech Effect: The role of short-term memory capacity. (in preparation)

Wightman, F.L., Kistler, D.J., O'Bryan, A. (2010). Individual differences and age effects in a dichotic informational masking paradigm. Journal of the Acoustical Society of America.128, 270-279.

\section{PRESENTATIONS}

O'Bryan, A. (December 2010). Individual Differences in susceptibility to the ISE. Invited talk given at the Speech Research Lab, Indiana University, Bloomington, IN

O'Bryan, A. (November 2010). The impact of short-term memory capacity on the Irrelevant Speech Effect. Experimental Psychology Graduate Student Seminar, University of Louisville, KY.

Kistler, D.J., Wightman, F.L., O'Bryan, A. (November 2010), Developmental change in spatial release from informational masking. Poster presented at the Auditory Perception Cognition and Action Meeting, St. Louis, MO.

O'Bryan, A., Wightman, F.L., Kistler, D.K., Rothpletz, A.M. (March 2010). Spatial Release from Informational Masking in Normally Hearing Children. Poster presented at the Graduate Research Symposium, University of Louisville, KY. 
O'Bryan, A. (January 2010). The development of the perception of prosody in children with cochlear implants. Experimental Psychology Graduate Student Seminar, University of Louisville, KY.

O'Bryan, A., Kistler, K.J., Wightman, F.L., (November 2009). The Irrelevant Speech Effect and Children: Methodological Considerations. Poster presented the annual meeting of the Psychonomic Society, Boston, MA.

Wightman, F.L., Kistler, D.J., Rothpletz, A.M., O'Bryan, A. (February 2009).

Informational Masking in Children with Cochlear Implants. Poster presented at annual meeting of the Association for Research in Otolaryngology, Baltimore, MD.

\section{COMPUTER SKILLS}

- Statistical software: PASW (SPSS), AMOS, HLM, Excel

- Computer programming: Matlab

- Database management: Microsoft Access, Filemaker

- Citation management software: Mendeley, Endnote

\section{PROFESSIONAL MEMBERSHIPS}

- Association of Psychological Science

- American Psychological Association

- Society for the Teaching of Psychology

\section{REFERENCES}

- Dr. Pavel Zahorik, Psychology, University of Louisville

- Dr. Ann Rothpletz, Audiology, University of Louisville

- Dr. Paul DeMarco, Psychology, University of Louisville 